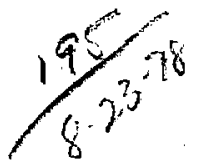

\title{
MASTER
}

16. 387

\section{AN EXPERIMENTAL STUDY OF THE USE OF BETHE HOLE THEORY FOR WIRES BEHIND APERTURES}

J. N. Brittingham

F. J. Deadrick

D. L. Lager

March 23, 1978

Work performed under the auspices of the U.S. Department of Energy by the UCLLL under contract number W-7405-ENG-48.

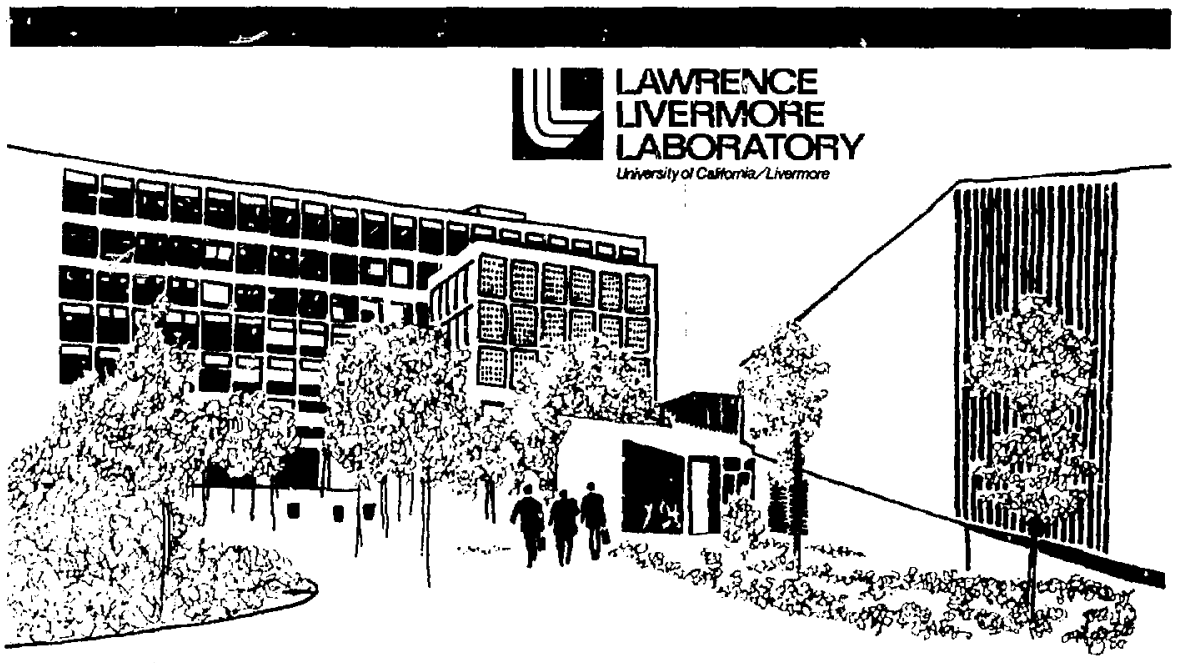




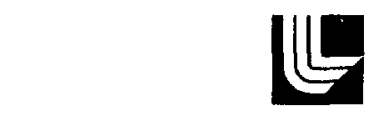

\section{LAWRENCE LIVERMORE LABORATORY}

University of Cattornia Livermore, Calilornia, 94550

UCRL -52443

\section{AN EXPERIMENTAL STUDY OF THE USE OF BETHE HOLE THEORY FOR WIRES BEHIND APERTURES}

J. N. Brittingham

F. J. Deadrick

D. L. Lager

MS. date: March 23, 1978 


\section{CONTENTS}

Abstract $\ldots \ldots \ldots \ldots \ldots \ldots \ldots \ldots \ldots \ldots \ldots \ldots \ldots \ldots \ldots \ldots$

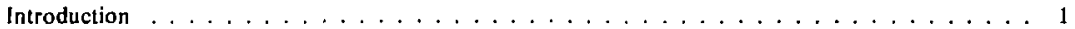

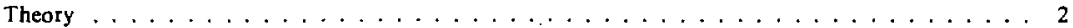

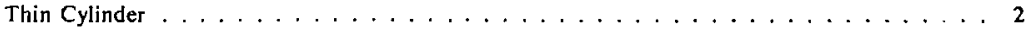

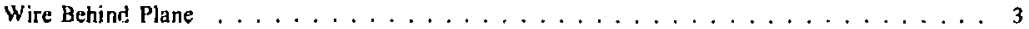

Fat Cylinder $\ldots \ldots \ldots \ldots \ldots \ldots \ldots \ldots \ldots \ldots \ldots \ldots$

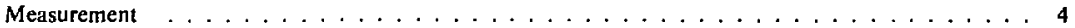

Geometric Description . . . . . . . . . . . . . . . . . . . 9

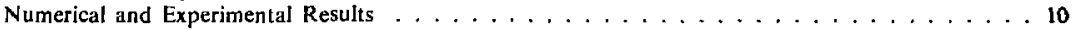

Discussion of Results . . . . . . . . . . . . . . . . . . . . . . . 54

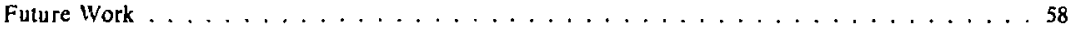

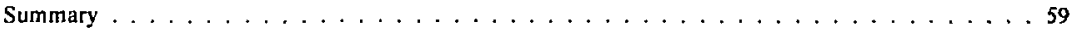

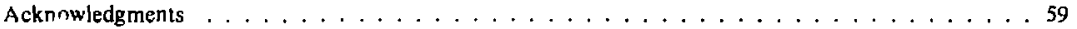

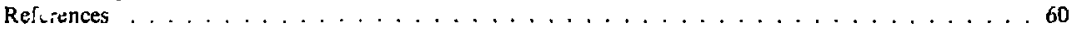




\title{
AN EXPERIMENTAL STUDY OF THE USE OF BETHE HOLE THEORY FOR WIRES BEHIND APERTURES
}

\begin{abstract}
This report presents an experimental validation of the use of Bethe hole theory in the numerical modeling of apertures in structures with wires behind the apertures. Three configurations are examined in this study: thin, capped cylinders with the wire located along the cylinder center; apertures in a plane with a wire; and fat, capped cylinders with wire inside. Currently accepted methods that use the Bethe hole theory to model the aperture are used to numerically model the current on the wires. The numerically generated current data are compared with experimental results. This validation is performed for both electrically small and large apertures.
\end{abstract}

\section{INTRODUCTION}

Over the last 90 years, many techniques have been developed for analyzing the coupling of electromagnetic energy through apertures. (See Ref. 1 for a review of these techniques.) Most of these methods have the limitation that the aperture must be electrically small or that the regions on either side of the aperture must be isolated from each other. These methods are often used on electrically large apertures, with possibly invalid results.

To validate all cases of coupling through apertures in practical electromagnetic pulse (EMP) problems would be an enormous undertaking. Since such a complete validation study is not practical, we limit this study to a few special cases to obtain some measure of the worth of using Bethe hole theory. The validation consists of comparisons between numerical and experimental results.

Three configurations are used in this study:

- Aperture in an electrically thin, finite-length, capped cylinder with a loaded wire at the center of the cylincie:

- Aperture in an infinite plane with a loaded wire behind the plane.

- Aperture in an electrically large, finile-length, capped cylinder with a loaded wire at the center of the cylinder.

Since the measurements are performed over a perfectly conducting ground plane, the cylinders and plane are connected to this ground plane. The wire inside the cylinder is open-circuited at the top of the cylinder and loaded at the end where the cylinder and ground plane meet. The apertures are cut at the point where the cylinder and ground plane meet. The plane that will later contain the aperture is placed at right angles to the ground plane. The wire behind the aperture perpendicular to the ground plane and a given distance behind the plane. The base of wire behind the plane is loaded with a resistance. The aperture is cut into the plane at the seam between the two planes. A plane wave is incident on the cylinders and plane from broadside, with the incident electrical field parallel to the central axis of the wire. The two problems are sketched in Figs. $\mathbf{i}$ and 2. This report begins with a discussion

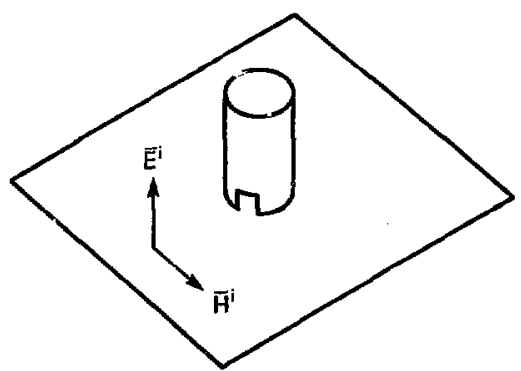

Fig. 1. Cylinder with aperture conmeeted to an infinite ground plane. An incideat plase wave excites the cyllnder. A whe is on the center of the cylinder. The top of the wire is eot electrically coneceted to the cylinder whlle the bottom of the wire is conaected to a So-n conxial transmiscion. In turn, the conxial cable is conarected to sampling scope wh a $50-\Omega$ input impedance. 


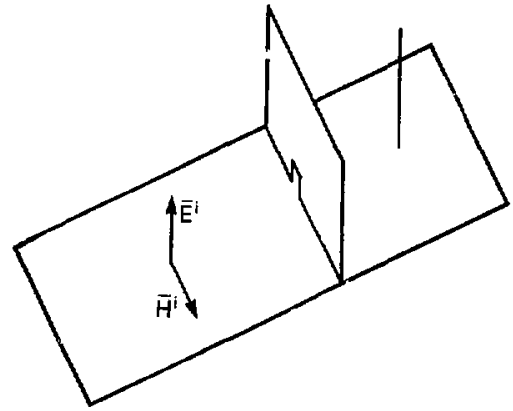

Fig. 2. Plane and aperture connected to an inlenite ground plane. An incident plane wave excites the cylinder. A wire perpendikular to the ground plane is connected to $50-\Omega$ coaxial transmission line. In turn, the coaxial transmission line is connected to a sampling scope with a $50-\Omega$ impedance. of the theory used to numerically model the problems followed by a description of the methods used to make the measurements. Numerical and experimental results are then presented along with a discussion of these results.

\section{THEORY}

\section{Thin Cylinder}

Thin cylinde ; are modeled by a procedure described by $\mathrm{Ta}_{\text {; }}$.or and Harrison. ${ }^{2}$ This procedure consists of first finding the current on the outside surface of the cylinder before the aperture is cut in to the cylinder. The magnetic field on the surface of the cylinder is then found from this current. This unperturbed magnetic field is assumed to be the magnetic field at the center of the apertuie when the aperture is placed in the cylinder. Bethe hole theory ${ }^{3}$ is then used to find the equivalent electric and magnetic dipole moment on the inside surface of the aperture These dipole sources are then assumed to couple into a coaxial transmission line inside the cylinder.

Before solving for the current on the cylinder, we find an equivalent problem for a cylinder connected to a perfectly conducting ground plane excited by a plane wave. First consider the cylinder and plane when the plane wave is incident from above the ground plane. This problem is depicted in Fig. 3a, where the angle $\phi$ is the angle of arrival for the incident plane wave. Figure $3 b$ depicts the equivalent problem when the greund plane has been removed. In this case, a second source had to be added along with the image of the cylinder below the interface. The second source is incident at an angle below the line where the interface had once existed. Figure 3c shows the results when the incident plane wave is allowed to become broadside, i.e., $\phi=0^{\circ}$. Note that the incident electric and magnetic fields are twice the values of the original fields. Therefore, when a plane wave is incident on a cylinder above a ground plane, the currents on the cylinder can be found by doubling the results for the equivalent problem involving the cylinder and its image. We now need only to calculate the current for the free space equivalent problem.

The current on the exterior of the thin cylinder is found by combining two solutions to the problem. The closed cylinder of finite length is first modeled by the thin-wire Numerical Electromagnetic Code* (NEC). ${ }^{4}$ Added to the current from this code is the niagnetostatic term investigated by Sancer. ${ }^{5}$ Figure 4 shows the external problem with a coordinate system. The height and radius of the cylinder are $L$ and $a$, respectively. Let the axia! current from the thin wire calculation at the point where the aperture is later placed be expressed as $I_{w}$. Assuming that this current is distributed uniformly around the surface of the wire, the z-directed current density on the surface of the cylinder is

*NEC is a moment-method computer program that can be used for modeling thin-wire problems (see Ref. 4). It also has a surface-patch capability to allow the modeling of solid bodies. 
(a)

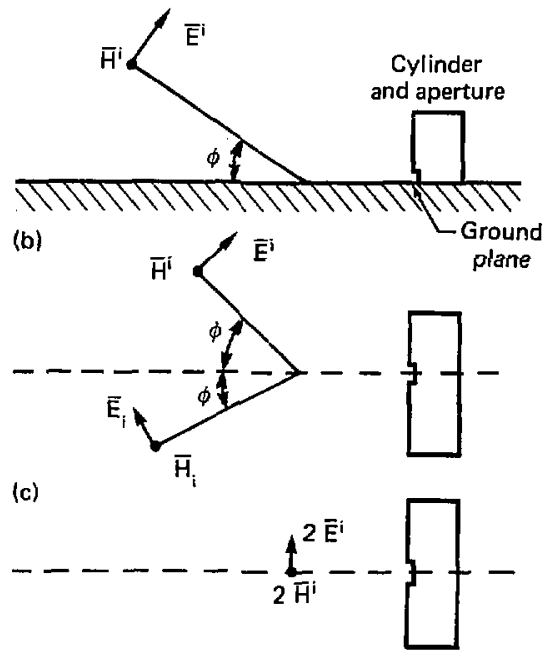

Fig. 3. The problem of a cylinder, aperture and ground plane (a) excited by plane wave; the equivalent problem with the ground plane removed (b); and the equivalent problem when the angle of the incident plane wave equals zero (c).

$$
J_{2}^{w}=\frac{I_{w}}{2 \pi a}
$$

The magnetostatic current density on a cylinder has been shown by Sancer ${ }^{5}$ to be

$$
\mathrm{J}_{\mathrm{z}}^{\mathrm{m}}=2 \hat{\mathrm{u}}_{\mathrm{z}} \cdot\left(\hat{\mathrm{n}} \times \overline{\mathrm{H}}^{\mathrm{i}}\right)
$$

where $\hat{\mathrm{u}}_{\mathrm{z}}$ and $\hat{\mathrm{n}}$ are the unit vectors representing the axial and normal directions. The total current density for the cylinder on the cylinder is

$$
\mathrm{J}_{\mathrm{z}}^{\mathrm{T}}=\frac{\mathrm{l}_{\mathrm{w}}}{2 \pi \mathrm{a}}+2 \hat{\mathrm{u}}_{\mathrm{z}} \cdot\left(\hat{\mathrm{n}} \times \overline{\mathrm{H}}^{\mathrm{i}}\right)
$$

Since $\mathbf{J}=\hat{\mathbf{n}} \times \overline{\mathbf{H}}$ on a perfectly conducting surface, the magnetic field on the cylinder prior to introducing the aperture is

$$
\mathrm{H}_{a}^{T}=\frac{1_{w}}{2 \pi \mathrm{a}}+2 \hat{\hat{u}}_{\mathrm{z}} \cdot\left(\hat{\mathrm{n}} \times \overline{\mathrm{H}}^{\mathrm{i}}\right) \text {. }
$$

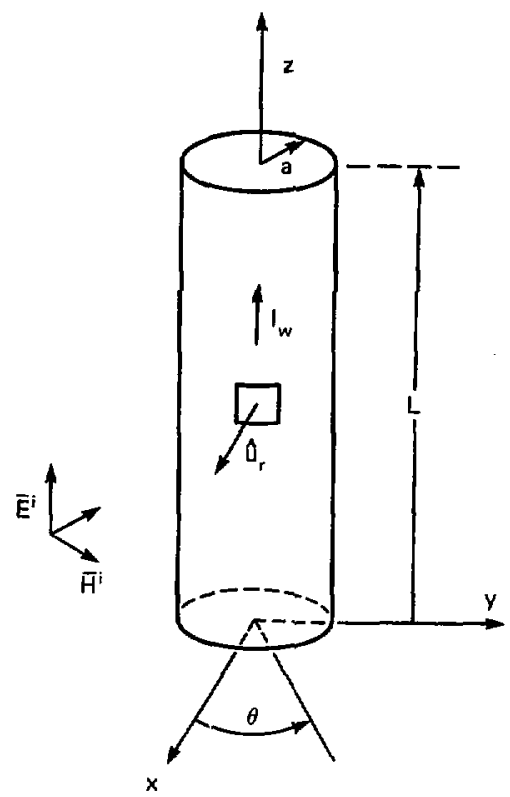

Fig. 4. Cylinder with aperture in free space.

where $\theta$ refers to the $\theta$ component of the magnetic field (see Fig. 4). The magnetic field expressed in Eq. (4) is the magnetic field at the surface of a closed cylinder in free space due to an incident plane wave. The free-space results expressed in Eq. (4) must be multiplied by 2 to find the magneti: field for the case of the cylinder above a ground plane. Al this point the magnetic field on the closed cylinder is assumed to be the sam; as when the aperture is placed in the cylinder.

With knowledge of tine mąnetic field in the aperture, we now use Bethe hore theory to find the equivalent source on the inside surface. The Bethe hole theory is a procedure by which the internal and external coupling of an aperture problem are isolated. The internal problem is handled by replacing the aperture with dipole sources. In this procedure the aperture is assumed to bc on a plane and the dimensions of the aperture are $k_{o} A_{m} r<1$, where $k_{o}$ is the free-space propagation constant and $A_{m}$ is the maximum dimension of the aperture. These reports neglect these limitations and uses the Bethe hole theory to model the aperture in the cylinder. We also push the theory beyond its limits when 
we use it to calculate the effect of the electrically large aperture.

The coupling coefficients for the Bethe hole theory are deveioped by Collin. ${ }^{3}$ The general Bethe hole theory has three equivalent dipole sources behind the aperture: an electric dipole source due to the normal component of the incident electric field through the aperture and two magnetic dipole sources due to the tangential components of the incivent magnetic field. In this study, we are considering a cylinder on a ground plane excited by a plane wave propagating along the ground plane. This form of excitation has no normal components of electric fields and only one component of the tangent magnetic fields in the aperture. This in turn gives us only a magnetic dipole source in the apertıre. Figure 5 depicts an aperture used in the Bethe hole theory along with local coordinate systems. By using Collin, ${ }^{3}$ the equivalent magnetic source on the inside of the cylinter is

$$
M_{\mathrm{ox}}=\iota^{\mathrm{K}} \mathrm{H}_{\mathrm{x}} \text {, }
$$

where

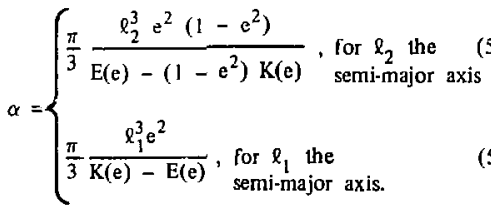

In Eq. (5b), $\ell_{1}$ and $\ell_{2}$ represent the semimajor and semiminor axes, respectively, of an ellipse inscribing the aperture, while in Eq. (5c), the notation is reversed. The eccentricity of the ellipse is

$$
e=\left[1-\left(\frac{\text { minor axis }}{\text { major axis }}\right)^{2}\right]^{1 / 2}
$$

The terms $E$ and $K$ are the elliptic integral of the first and second kind, respectively. The magnetic field of Eq. (5a) is the field at the center of the aperture. As noted above, the magnetic field for a cylinder in free space must be multiplied by 2 to find the magnetic field for a cylinder and ground plane. Therefore, the magnetic field used in Eq. (5a) must be twice the field presented in Eq. (4).

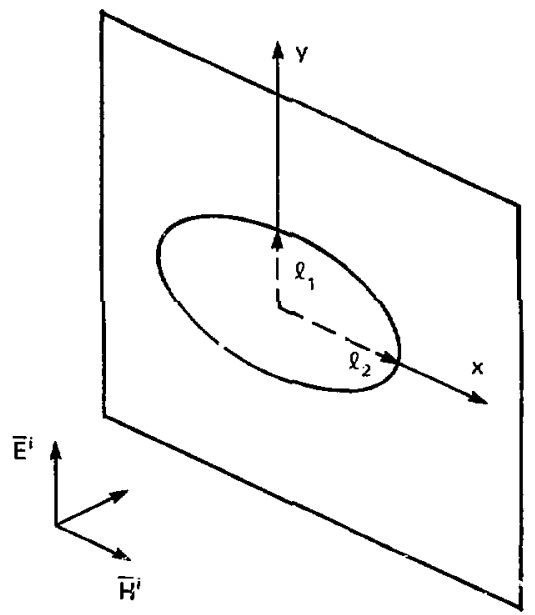

Fig. 5. A perture in a plate of infinite extent.

Now that the dipole moment for the aperture is known, we can solve for the current at the base of the center conductor. 1 sketch of this problem is shown in Fig. 6. Since only half the cylinder is used in this figure, only half the dipole source expressed in Ey. (5a) is needed to describe the source. By using a procedure similar to that presented by Taylor and Harrison, ${ }^{2}$ which allows a magnetic dipole source to couple into a coaxial transmission line, we obtain the load current:

$$
\mathrm{I}_{\mathrm{L}}=\frac{j \omega \mu_{\mathrm{o}} \mathrm{M}_{\mathrm{ox}}}{4 \pi \mathrm{b}}\left(\frac{1}{\mathrm{Z}_{\mathrm{s}}^{\prime}}\right)
$$

where

$$
Z_{s}^{\prime}=R_{L}+Z_{c}\left[\frac{1}{\tanh (\gamma L / 2)}\right]
$$

The terms $b, r_{w}, L / 2$, and $R_{L}$ are the interior radius of the cylinder, the wire radius, length of the wire, and load resistance, respectively. The propagation constant for a coaxial line can be written as

$$
\gamma=\mathrm{j} \omega / \mathrm{c},
$$




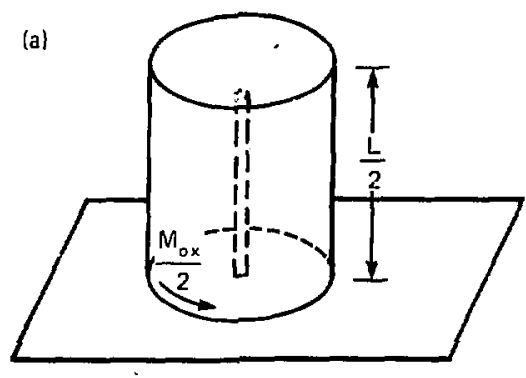

(b)

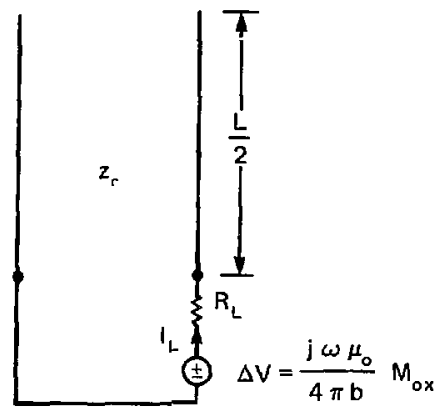

Fig. 6. Cylinder with a wire at the center excited by a magnetic dipole source on the inside surface of the cylinder ( $\mathrm{a}$ ). Also shom is the equivalent transmissiondine analogue (b). The $\mathbf{R}_{\mathbf{L}}$ in the equiralent problem is the input inpedance of the sampting scope used to measure the current at the base of the wire.

where $\mathrm{c}$ is the propagation velocity in the transmission line. The characteristic impedance of a coaxial line is

$$
Z_{c}=60 \ln \left(b / r_{w}\right)
$$

The parameters w and $\psi_{0}$ are assumed to be angular frequency and the magnetic permeability of free space, respectively. in Eqs. (7a) and (7b), the condition that the end of the wire is not electrically connected to the end of the cylinder has been used in the derivation of these results.

\section{Wire Behind Plane}

By using an argument similar to that used for the thin cylinder, we can show that two planes at right angles to each other with an aperture in one can be represented as a single plane in free space. In the equivalent problem, the incident fields are doubled and an image is introduced. The numerical modeling of a wire behir.d a plane with an aperture in the plane uses a procedure derived by $\mathrm{Kajfez} .{ }^{6}$ In this procedure he first finds the dipole currents on the backside of the aperture by Bethe hole theory. These dipole sources are then coupled into the twowire transmission line formed by the wire and its image in the infinite conducting plane.

Since the incident field is a plane wave that strikes the aperiure broadside, only tangential incidem fields are in the aperture. The tangential magnetic field gives rise to a magnetic dipole source on the inside surface of the aperture, while the electric field has no current source associated with it. The magnetic dipole source can be expressed by using Eq. (5a). The magnetic field used in this equation is twice the magnetic field of the original plane wave. Since the definition of magnelic dipole moment used by Collin ${ }^{3}$ is diff-rent from that used by Kajfez, ${ }^{6}$ the magi.etic dipole moment in Eq. (5a) must be multiplied by jw/ $\mu_{0}$ when used in Kajfez's formulation.

The equivalent problem of a magnetic dipole source on a plane with a wire behind the plane is shown in Fig. 7a. When the ground plane is replaced by the wire and its imaze, the dipole moment exciting the whole space behind the aperture is $M_{o x}$, but when the ground plane is left in the problem, the dipole moment behind the aperture must be divided by 2 . The transmission-line equivalent of this problem is shown in Fig. $7 \mathrm{~b}$.

By using Eq. (C-15) in Ref. 6, the equivalent transmission line sources can be expressed as

$$
\Delta \mathrm{J}=\mathrm{D}
$$

and

$$
\Delta Y=\frac{\left(j \omega \mu_{\mathrm{o}}\right) M_{\mathrm{OX}}}{\pi \sqrt{\mathrm{d}_{1}^{2}-\mathrm{r}_{w}^{2}}} .
$$

where $d_{1}$ and $r_{w}$ are the distance from the center of the wire to the conducting plane and the radius of the wire, respectively. The impedance looking up from the source in Fig. $7 \mathrm{~b}$ is

$$
\mathrm{Z}_{1}^{\mathrm{P}}=\mathrm{R}_{\mathrm{L}}+\mathrm{Z}_{0}^{\prime}\left[\frac{1}{\tanh (\gamma \mathrm{h} / 2)}\right]
$$


(a)

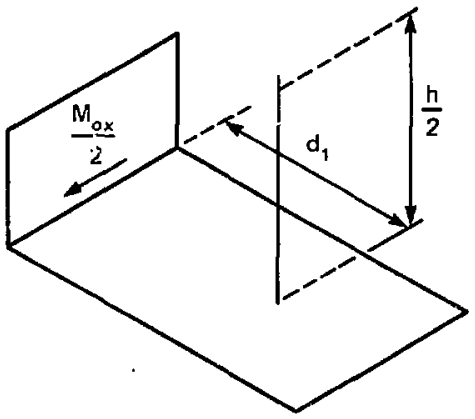

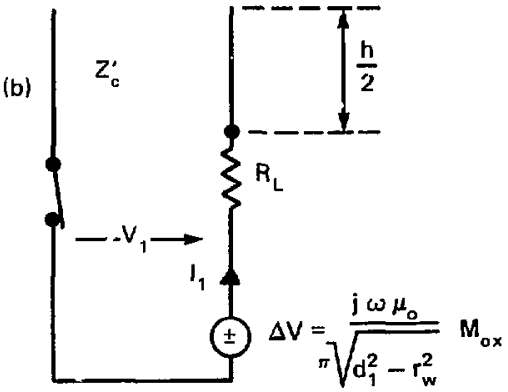

Fig. 7. Two infinite planes and wire excited by a magnetic dipole source at the point where the two planes meet (a). Also shown is the equivalent trangmission-line analogue $(b)$. The $R_{L}$ in the equivalent problem is the input impedance of the sampling scope used to measure the current at the base of the wire.

where the characteristic impedance for a two-wire transmission line is

$$
Z_{0}^{\prime}=60 \cosh ^{-1} \frac{d_{1}}{I_{w}} .
$$

Using the fact that

$$
I_{1}=\frac{v_{1}}{Z_{1}^{p}}
$$

along with Eqs. (9)-(11), we can show

$$
I_{1}=\frac{1}{\pi} \frac{j \omega \mu_{o} M_{o x}}{\sqrt{d_{1}^{2}-r_{w}^{2}}} \frac{1}{Z_{1}^{p}} .
$$

The $1_{1}$ above is the current in the load.

\section{Fat Cylinder}

The electrically fat cylinders are modeled much the same way as the thin cylinder, except for the method used to find the currents on the outside surface of the cylinder before the aperture is placed into the cylinder. For this case, the fat cylinder is numerically modeled by using the patch capability of $\mathrm{NEC}^{4}$ on the conducting surface of the cylinder. This procedure consists of representing the surface of the cylinder by patches and solving the magneticfield integral equation using collocation with pulsebasis functions and Dirac delta-function weights. Once the .."rrent density on the outside surface of the cylinder is known, the equation $\bar{J}=\hat{n} \times \bar{H}$ is used to show that the magnetic field is

$$
-T_{6}=J_{2}
$$

Note that Eqs. (4) and (15) are different in that Eq. (4) has a magnetostatic term added to the thinwire results, while Eq. (15) has the current density from the paich solution to fat cylinder, which, of course, is a complete description of surface-current density. From this point the solution to the fat cylinder is handled identically to the thin cylinder presented above.

\section{MEASUREMENT}

The measurements of the three structures were performed on the Lawrence Livermore Laboratory (LLL) Tr:asient Electromagnetic Facility (see
Deadrick et at. ${ }^{7}$ for a description). The facility consists of a ground plane with a cone antenna. Aluminum plates are electrically connected to form 
a conducting surface $8.5 \times 8.5 \mathrm{~m}$ that is $0.091588 \mathrm{~m}$ thick. Connected to the center of the ground plane is a conical antenna whose vortex is at the center of the ground plane. The vertex of the conical antenna is excited by an IKOR IMP* impuise generator. The pulsp .om the generator flas a Gaussianshaped wi:"eform with a 300.0 ps pulse width and a $1.0-\mathrm{kV}$ peak. The conical anteana and its image in the ground plane produce plane-wavelike fields rear the ground plane.

To obtain the cylitider nieasurements, apertures are placed in the base of the copper cyliniter, which is then soldered to the ground plane. $\dot{A}$ copper cap is placed on the top of the cylinder. At the point where the center of the cylinder crosses the ground plane, an electrical connector for coaxial transnission lines is placed through the ground plane. A copper wire is placed in the center of the connector and extended the full length of the cylincer. This wire is not electrically connected to the top of the cylinder. To measure the current at the base $G$ a the wire, a $50-\Omega$ coaxial cabie (Tektronix-SMA $.003 \mathrm{~m}$ ) is connected to the electrical connector under the ground $\mathrm{f}$ ne. This coaxial cable is connected to a sanupling scope with an input impedance of $50 \Omega$.

The aperture-in-Flane measurements are made by constructing a fine-mesh ( 80 wirs $/$ in.), 8.5 -m-wideby-2,24-m-high copper screen perpendicular to the ground plane of the iange. A wooden frame supports the screen from behind (see Fig. 8). Where it meets the ground plane, the screet has a right-angle metallic lip that makes good electrical contact with

\footnotetext{
- Reference to a company or product name does not imply approval or recommendation of the product by the University of California or the U.S. Energy Rescarch \& Development Administration to the exclusion of others that may be suitable.
}

the ground plane. 1 large bole is cut into the center of the screen where the screen and ground plane meet. An aluminum plate $.91 \times .91$ and $0.001588 \mathrm{~m}$ thick is placed over the hole . : the ssreen. This plate, with the copper screen beneath, is bolted to ihe woosen frame to ensurc good electrical contact between the screen and plate. Apertures are cui into this plat at the point where the piate metts the ground plane. Along the edge where the plate and ground plane meet, electrical tape is used to guarantee good electrical contact on the iront side of the plate. To support the wire b-nind the aperture, a coaxial connector is placed through the ground plane of the range at the appropriate distance behind the aperture. A vertical wire is placed into the center of the connector. The current at the base of the wire is measured by a sampling scope beiow the range with a $50-\Omega$ input impedance. The connertion between the base of the wire and sampling scope is made with $50 \Omega$ coaxial cable (Tektroni.i-SMA $0.003 \mathrm{~m}$ ).

The incident plane wave is measured before the cylinder or screen are placed on the range. $A \dot{D}$ sensor : $\triangle F W L A C D-1$ ) was used to measure the normal component of the time derivative or the electric flux density. The data from the $\dot{D}$-sensor are int egrated to give $E_{\mathrm{i}}(t)$. The incident electric field is fast-Fourier transformed to obtain the frequency response of the incident electric field. Later, the time response of the current is measured in the $50-\Omega$ transmission line connezted to the base of the wire behind the apertures. The time response of the current is also transformed ina a frequency resnonse by a fast Fourier transform. The transfer function for the aperture and wire is obtained by dividing the frequency response of wire and aperture by the frequency response of the incident planc wave. 

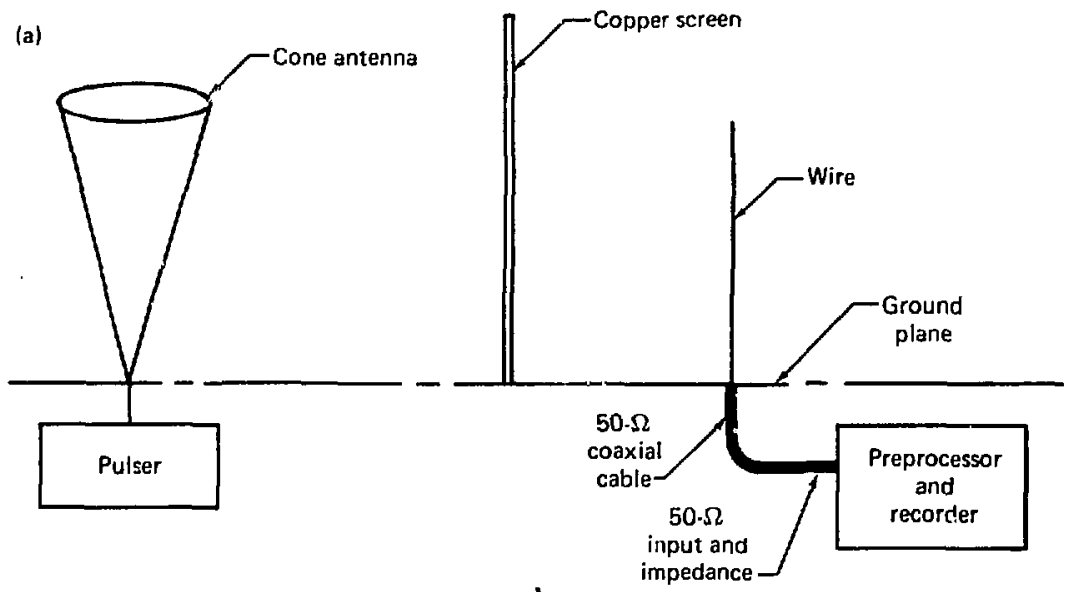

(b)

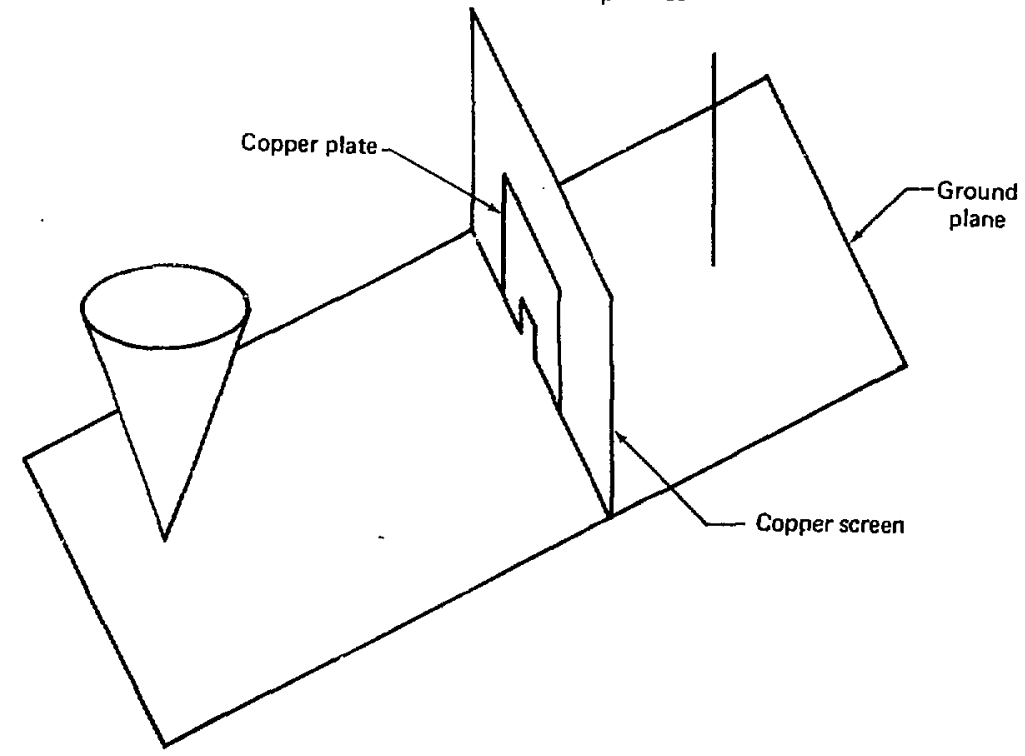

Fig. 8. Proslle (a) and perspective (i) vlews of a transient mensurement range and the conducting plane with apertures and wire. 


\section{GEOMETRIC DESCRIPTION}

The thin cylinder used in the numerical and experimental study has an outside ditumeter of $0.051 \mathrm{~m}$. an inside diameler of $0.049 \mathrm{~m}$, and a total length above the ground plane of $0.60 \mathrm{~m}$. The wire on the inside of the cylinder is $0.59 \mathrm{~m}$ long with a $0.0016-m$ diameter. The wire was not connected to the top of the cylinder. Three apertures were cut into the side of the cylinder. The sizes and shapes of these are given in Table 1 . The axial dimensions listed in Table 1 account for the fact that the aperture have an image in the ground plane, therefore the given lengths are twice the physical length of the apertures cut into the cylinder. Each aperture was run for three cases of incident plane wate: head-om, $90^{\circ}$ from head-on, and for the shaded side of the cylinder.
The wire used in the study of an aperture in a plane has a length of $0.5 \mathrm{~m}$ above the groind plane and a diameter of $0.0015 \mathrm{~m}$. The sizes and shopes of the apertures cut into the plane are presented in Table 2. For each aperture, the load current at the base of the wire was incasured at a wire distince of $0.05,0.35$, and $1.15 \mathrm{~m}$ behind the planc. Th: only exception to this was that the last distance is $0.95 \mathrm{~m}$ instead of $1.15 \mathrm{~m}$ for the last two apertures sho $\mathrm{vn}$ in Table 2. This measurement is performed or: a line that is perpendicular to the plane containing the aperture and that passes through the center of the aperture. The aperture heights listed in Table 2 are twice the physical lengths of the apertures cut into the plane, to account for the image of the aperture in the ground plane.

Table 1. Size and shape of aperture in thin cylinder study.

\begin{tabular}{lccl}
\hline Apet iure & $\begin{array}{c}\text { Ciscumferential } \\
\text { dimension, } \\
\mathrm{m}\end{array}$ & \multicolumn{1}{c}{ Description } \\
\hline 1 & 0.02 & 0.01 & Rectangular \\
2 & 0.06 & 0.01 & Axial slit \\
3 & 0.02 & 0.16 & $90^{\circ}$ Circumferential slit \\
\hline
\end{tabular}

Table 2. Size and shape of aperture in study of wire hehind plane.

\begin{tabular}{|c|c|c|c|}
\hline Aperture & $\begin{array}{c}\text { Metughe, } \\
\text { m }\end{array}$ & $\begin{array}{c}\text { width, } \\
\text { m }\end{array}$ & Description \\
\hline 1 & 0.16 & 0.16 & Circular \\
\hline 3 & 0.2 & 0.2 & Square \\
\hline 3 & a.os & 0.08 & Square \\
\hline 4 & 0.1 & 0.1 & Square \\
\hline 5 & 0.3 & 0.2 & Rectangular \\
\hline
\end{tabular}

The fat cylinder used in this study has an outside diameter of $0.255 \mathrm{~m}$, an inside diameter of $0.250 \mathrm{~m}$, and a total length of $0.23 \mathrm{~m}$ above the ground plane. The wire on the inside of the cylinder is $0.22 \mathrm{~m}$ long with a $0.0017-\mathrm{m}$ diameter. The wire is not connected to the top of the cylinder. The sizes and shapes of the apertures cut into the side of the cylinder are shown in Table 3. The aperture axial dimensions listed in Table 3 are twice the physical lengths of the apertures cut into the cylinder. Each aperture was run for three cases of the incident plane wave; head on, $90^{\circ}$ irom head-on, and for the shaded side of the cylinder.

Table 3. Size and shape of apertures in fat cylinder study.

\begin{tabular}{|c|c|c|c|}
\hline Aperture & $\begin{array}{c}\text { Axial dimension, } \\
\text { m }\end{array}$ & $\begin{array}{c}\text { Circumferential } \\
\text { dimension, } \\
m\end{array}$ & Description \\
\hline $\mathbf{1}$ & 0.10 & 0.10 & Circular \\
\hline 2 & 0.15 & 0.10 & Rectangulay \\
\hline 3 & 0.10 & 0.15 & Rectangular \\
\hline 4 & 0.30 & 0.05 & Axinl slit \\
\hline$s$ & 0.10 & 0.20 & $90^{\circ}$ Circumferentiv slit \\
\hline
\end{tabular}




\section{NUMERICAI، AND EXPERIMENTAL RESULTS}

In this section the numerical and experimental results obtained for $t$.e three shapes described in the previous section are presented. For convenience, all plots and remaining tables are located at the end of the report.

The results for the thin cylinder are presented first. These numerical results are partitioned into three scparate transfer functions. The first transfer function $T_{1}$ is used to obtain the current density on the outside surface of the cylinder due to an incident plane wave. The second transfer furction $T_{2}$ permits determination of a magnetic dipole current on the inside of the cylinder caused by the magnetic field in the aperiure, while the last transfer function $T_{3}$ provides the current at the base of the wire inside the cylinder that results from a magnetic dipole source on the inside surface of the cylinder when the ter $\mathrm{m} j \omega \mu_{o} / 4 \pi \mathrm{b}$ has been divided out. The expression for calculating $T_{1}$ is Eq. (3). The second transfer fu iction $T_{2}$ can be calculated by Eq. (5). The last transfer function $T_{3}$ is the term in the brackets of Eq. (7a). The iutal transfer function an be expressed as

$$
T_{T}=\frac{j \omega \mu_{0}}{4 \pi b} T_{1} T_{2} T_{3} .
$$

Plots 1-3 show the frequency plot of the normalized magnitude of current density $T_{1}$ at the point where the aperture will cut into the side of the thin c:linder for the cases where the incident plane wave is head on, $90^{\circ}$ from head-on. and backside of the cylinjer. Next, Table 4 (page 13) presents the values of the Beihe hole coefficient $T_{2}$ for the apertures cut into the thin cylinder [these apcriuses are listed in Table I (page 9)]. Plol 4 shuws the frequency plot

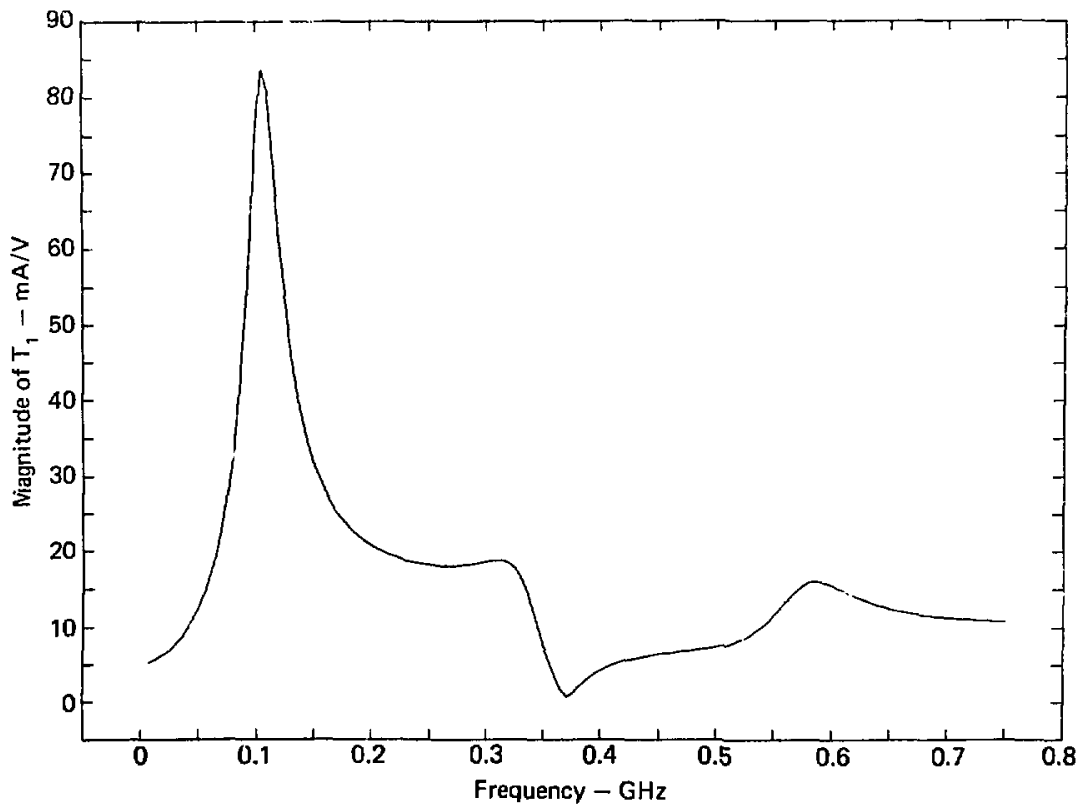

Plot. 1. Magnitude of the transfer function of the external current density at the center of an electrically thin cylinder from an incident plane mave, T . This current is calculated from Eq. (3). The point shown here is on the illuminated side of the cylinder. 


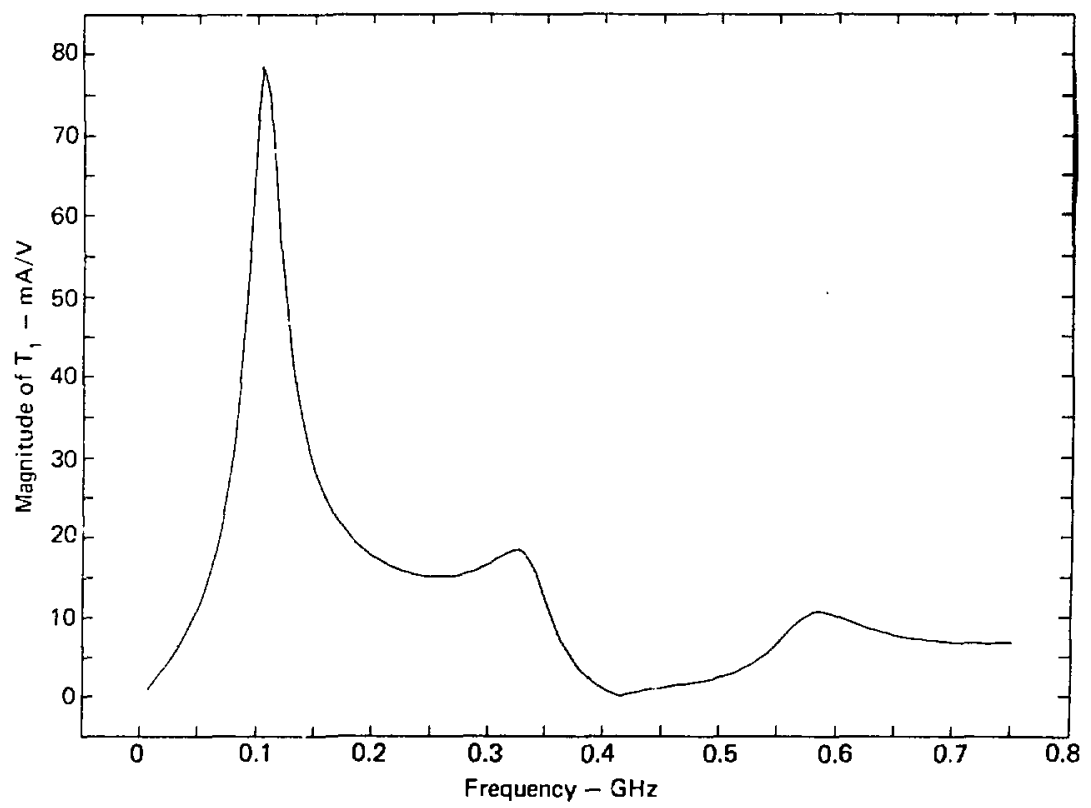

Plot 2. Same graph as Plot 1 , except the point on the cylinder is $90^{\circ}$ to head-on incident. 


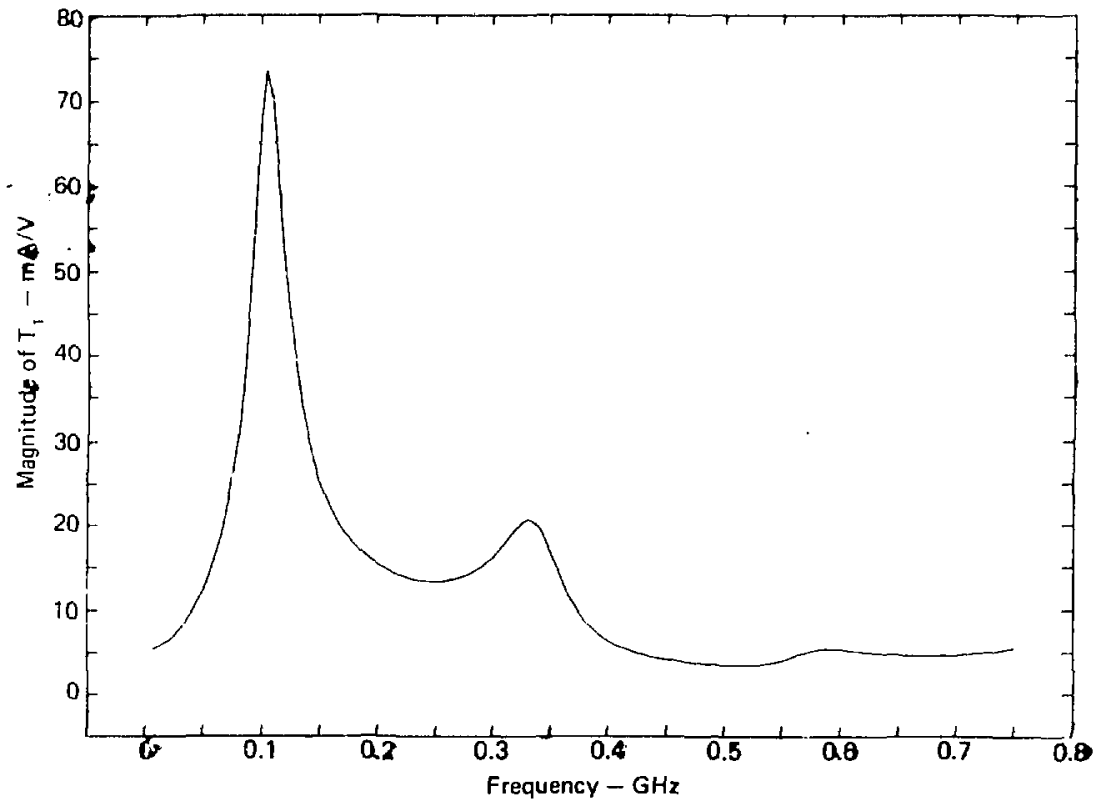

Plot 3. Sane graph as Phot 1 , except the polex is on the thaded sde of ibe cylinder. 
Table 4. Values of Bethe hole coefficient for aperture in the cylinder experiments."

\begin{tabular}{lll} 
Apertuse & $\begin{array}{c}\text { Diniensions, } \\
\text { nI }\end{array}$ \\
\hline 1 & $0.02 \times 0.01$ & $-2.921 \times 10^{-7}$ \\
2 & $0.06 \times 0.01$ & $-8.048 \times 10^{-7}$ \\
3 & $0.02 \times 0.16$ & $-2.152 \times 10^{-4}$ \\
\hline
\end{tabular}

These rewles were numerieally evalusted from Eqa. (56) and $(\mathrm{Sel}$.

of the transfer function $T_{3}$ used to determine the mignitude of current at the base of the wire resulting from a magnelic dipole source on the inside surfice of the cylinder. Plots 5-13 display the frequency plot for the normalized inagnitude of current at the base of the wire resulting from an incudent plane wave. These plots present both the numerical ans experimental scsults; the solid and dotied curves represent the numerical and ex. perimental data, respectively. These plots show the data for the three apestures and three different arrival angles of the incident plane wave.

The results for an aperture in a plane with a wire bein. nd the plane are presented next. In this study only the transfer function for the current at the base of the wire from an incident plane wave is given. The numerical model is calculated from Eq. (14) above. The magnitudes of the experimental and numerical transfer functions are shown in Plote $\mathbf{4}$ 28. These plots display the data for the casea of the five apertures and three wire-plane distances given in Table 2. The numerical and experimental curves are depicted by solid and cotted lines, respectively.

The results for the fat sylinder are presented in the sime partitioned format that was used to calculate the thin cylinder results. In the case of the fat cylinder, the last two transfer functions, $T_{2}$ and $T_{3}$, are calculated by the same formalation used for (Text conimues on page 38 .)

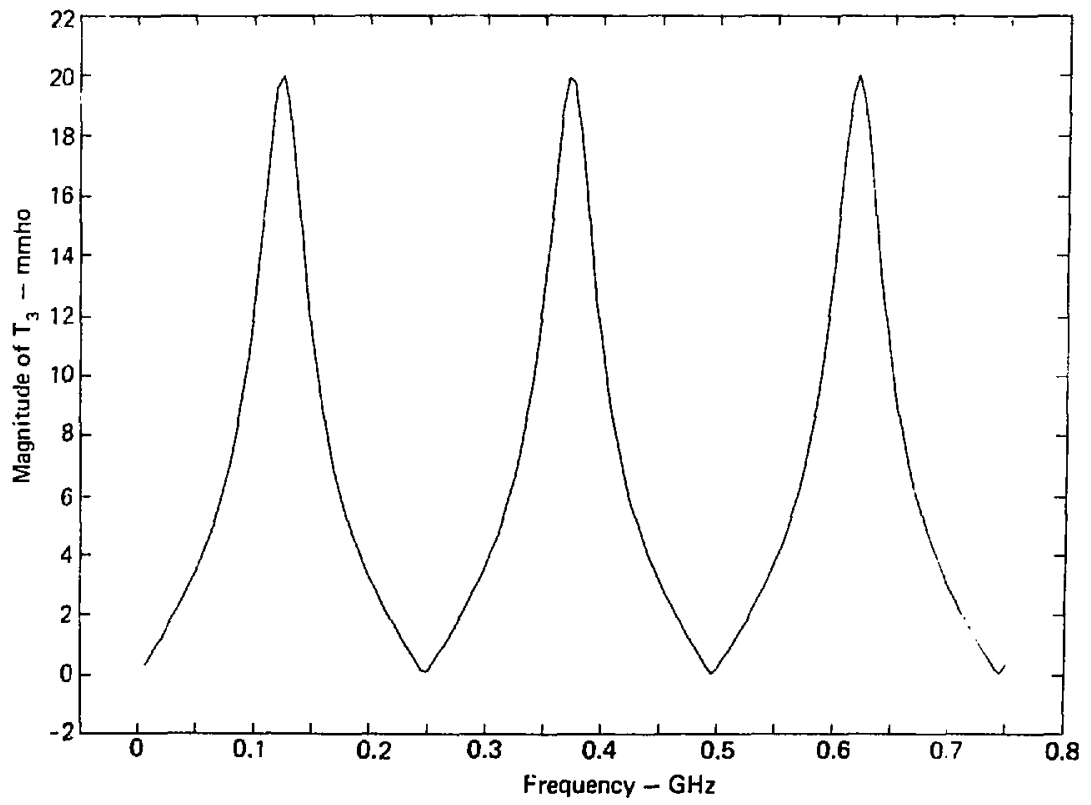

Plot 4. Magnitude of the transfer function of the current at the base of the wire from a magnetic dipolv source on the wurface of the thin cylinder, $T_{3}$. These results were numerically evaluated from the term inside the brackets of Eq. (7a). 


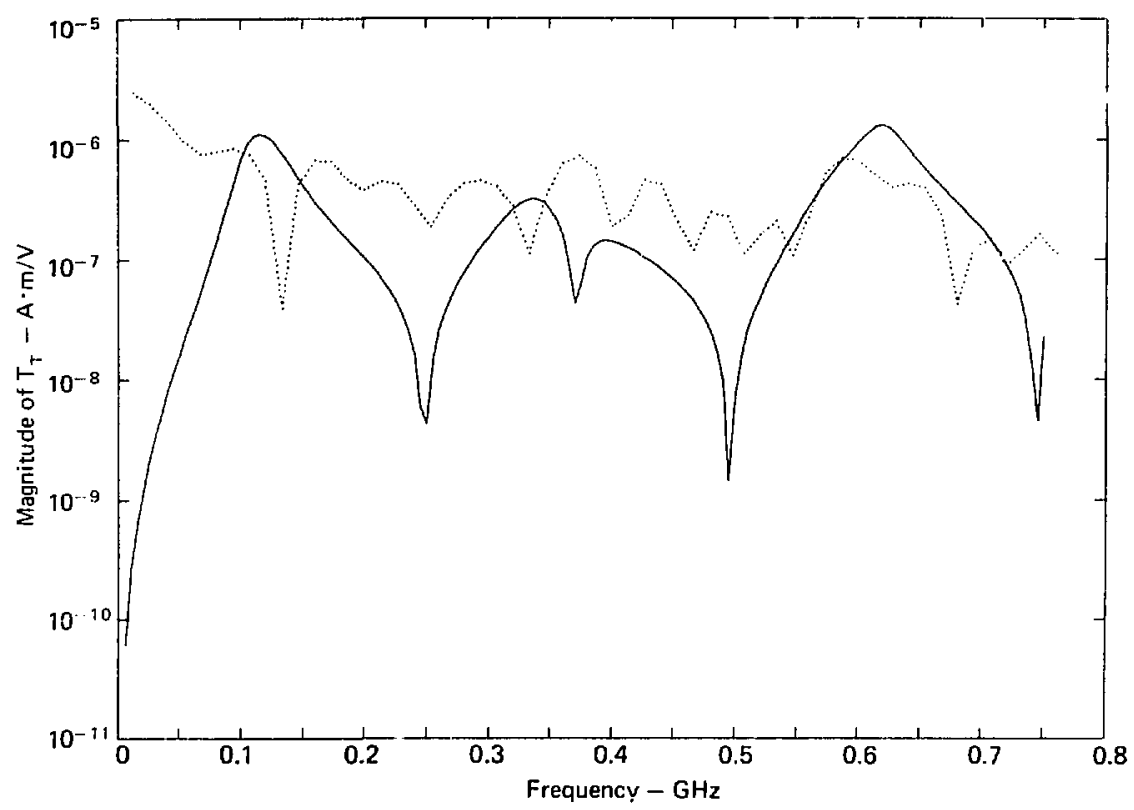

Plot 5. Magnitude of the transfer function of the current at the base of the wire from an incident plane wave on the thin cylinder, $T_{\mathrm{r}}$ In this plot the axial and the circumferentiat dimensions of the aperture are 0.02 and $0.01 \mathrm{~m}$, respectively. The aperture is on the illuminated side of the cylinder. The solid and dotted curves represent the numerical and experimental results, respectivsly. 


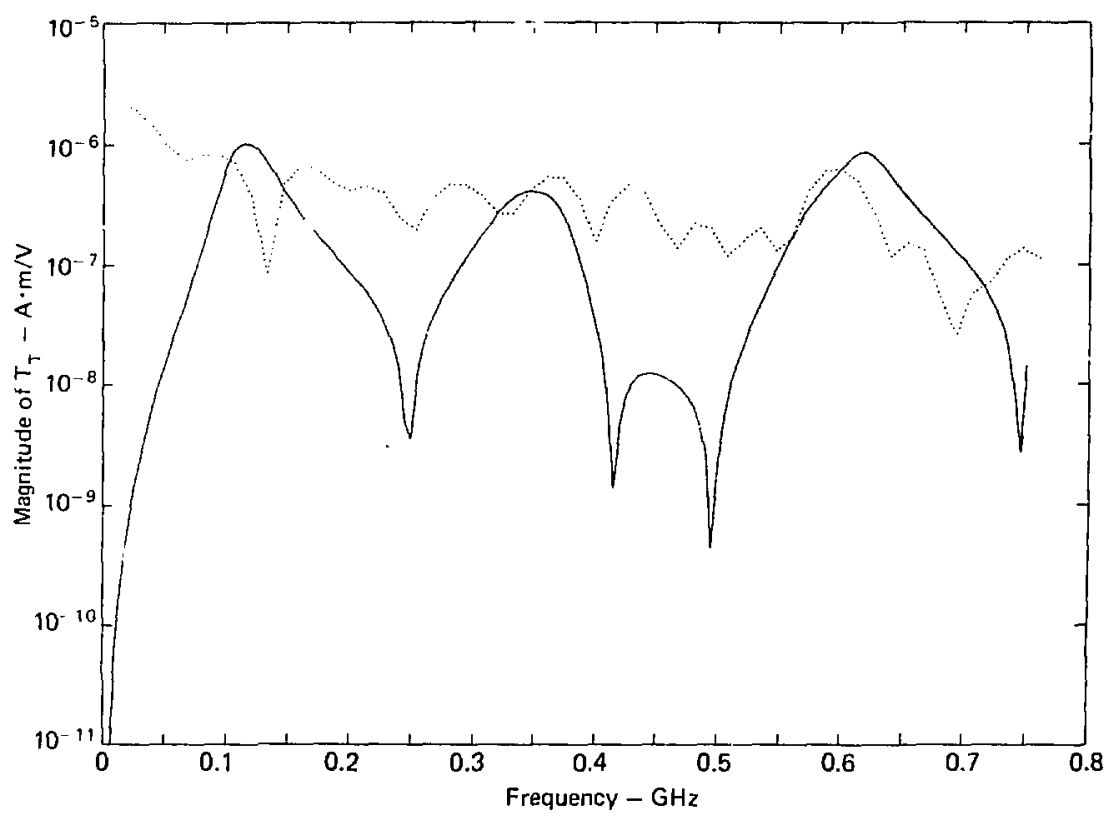

Plot 6. Same yraph as Plot 5 , excep1 the axial and the circumferential dimensions or the apertuze are 0.02 and $0.01 \mathrm{~m}$, zespertively. The aperture is on the cylinder $90^{\circ}$ from head-on incidence. 


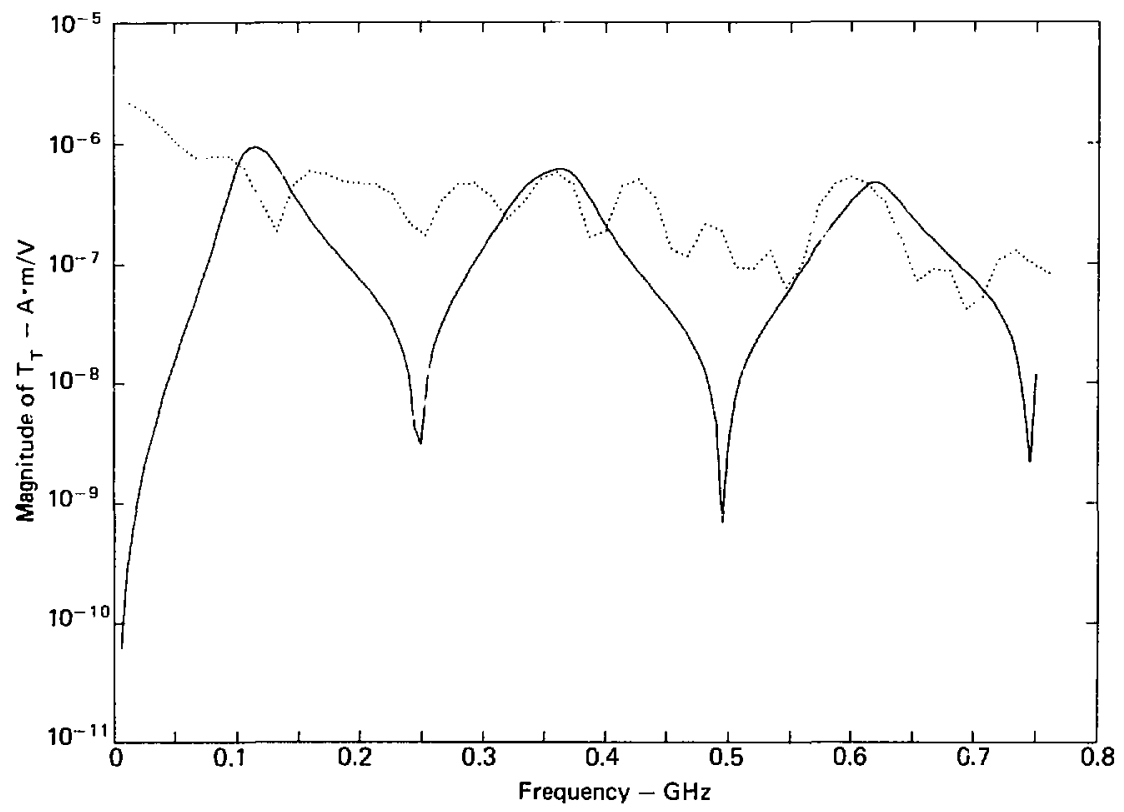

Plut 7. Same graph as Plot 5 , except the axial and circumferential dimensions of the aperture are 0.02 and $0.01 \mathrm{~m}$, respectively. The aperture is on the shaded side of the cylinder. 


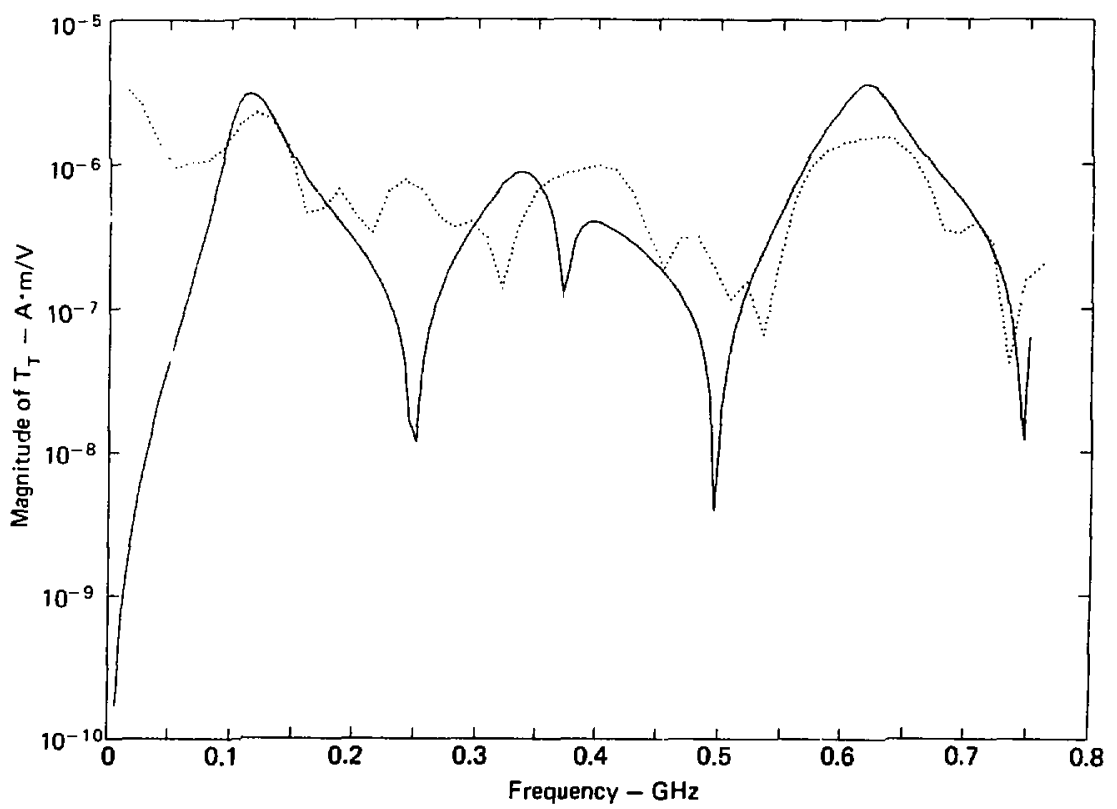

Plot 8. Same graph as Plot 5 , except the axial and circumferentisl dimensions of the aperture are 0.06 and $0.01 \mathrm{~m}$, respectively. The aperiure is on the illominated stide of the cylinder. 


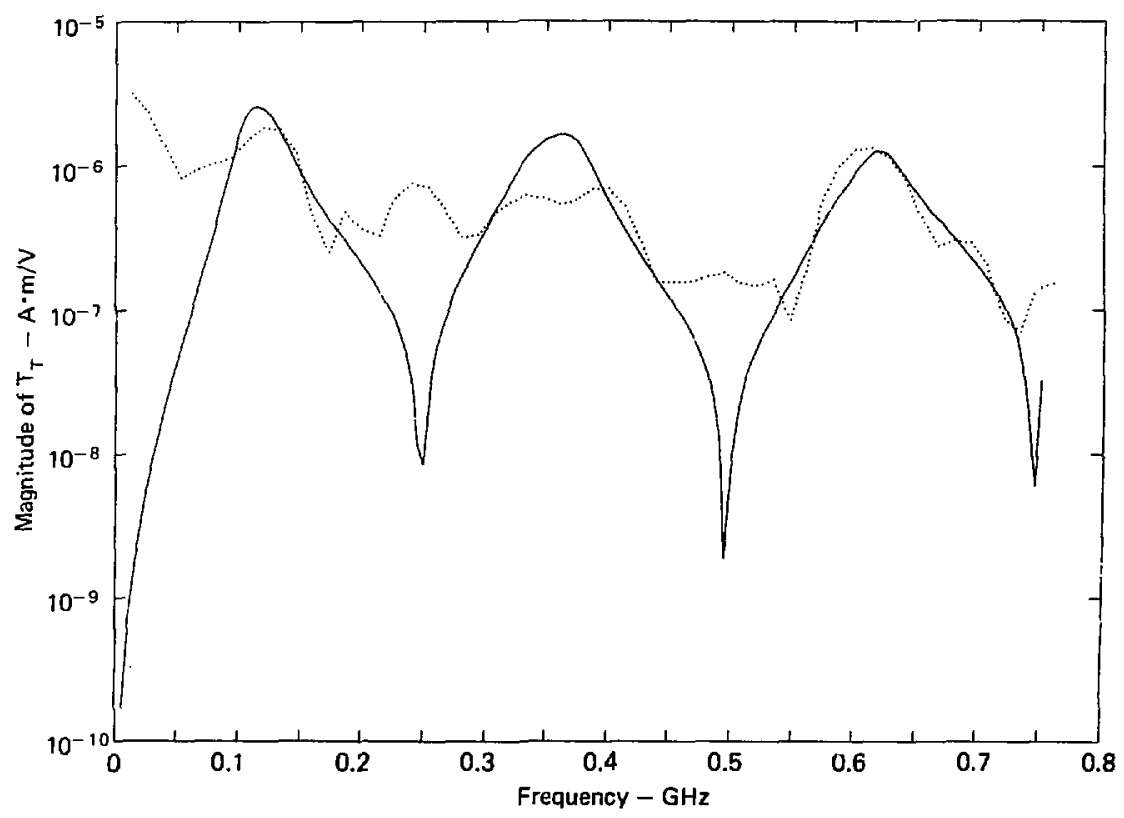

Plot 30. Same graph as Plot 5, except the axial and the circumferential dimensions of the aperture are 0.06 and 0.01 m, respectively. The aperture is on the shaded slde of the cylinder. 


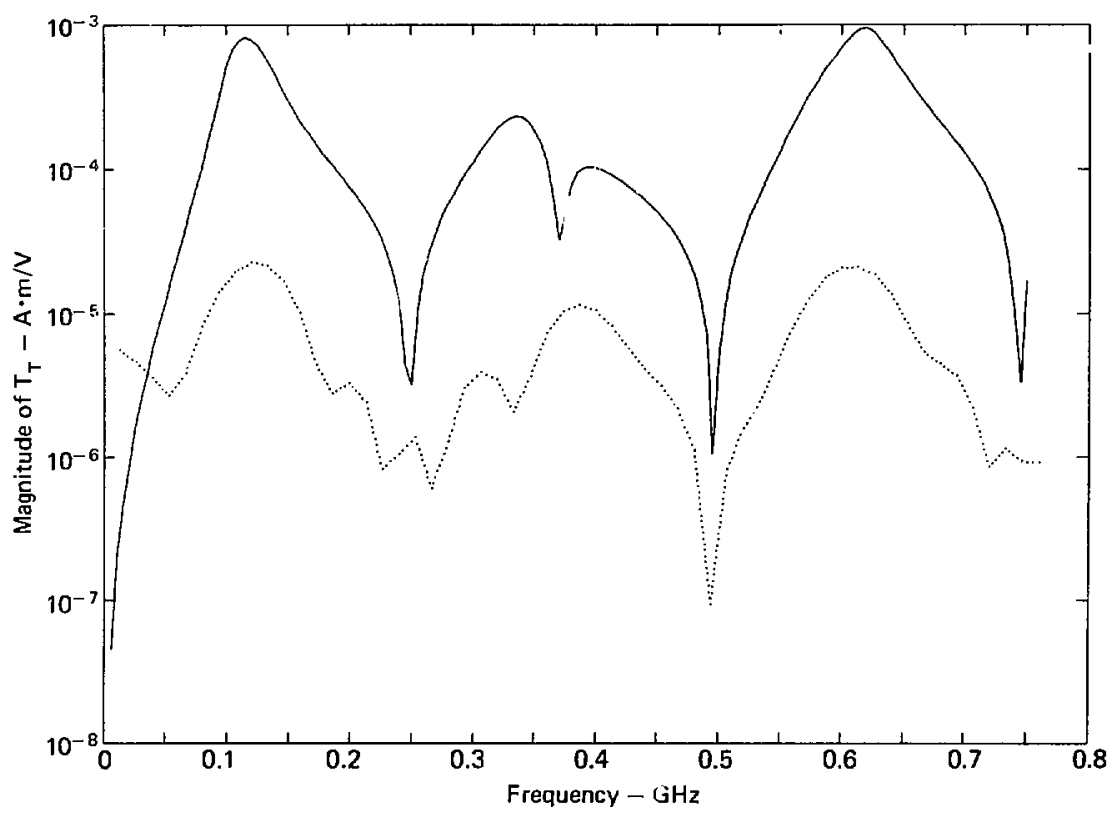

Plot 11. Same graph as Plot 5 , except the aperture is $q 90^{\circ}$ circumferential slit with an axint dimension of $0.02 \mathrm{~m}$. The aperture is on tine illuminated side of the cylinder. 


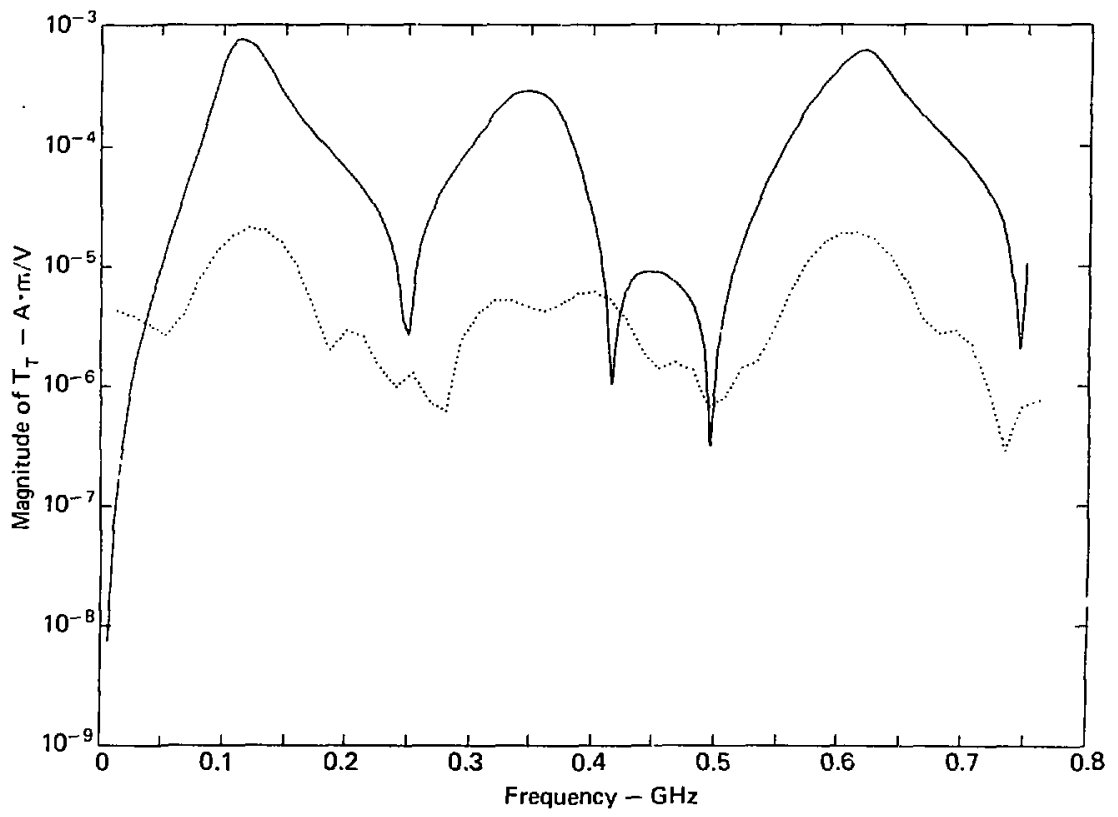

Plot 12. Same graph as Plot 5 , except the aperture is $a 90^{\circ}$ circumferential slit with an axial dimension of $0.02 \mathrm{~m}$. The aperture is on the cylinder $90^{\circ}$ from head-on incidence. 


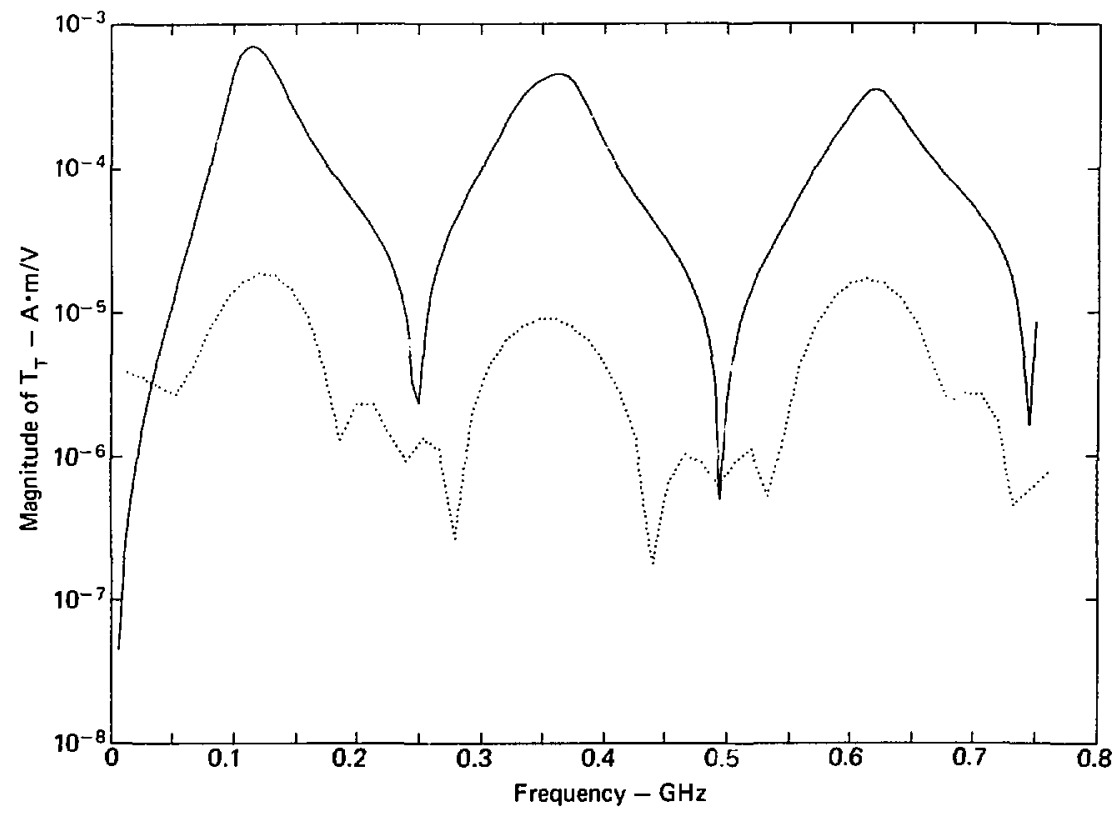

Plot 13. Same graph as Plot 5 , except the aperture is a $90^{\circ}$ circumferential slit with an axial dimension of $0.02 \mathrm{~m}$. The sperture is on the shaded side of the cylinder. 


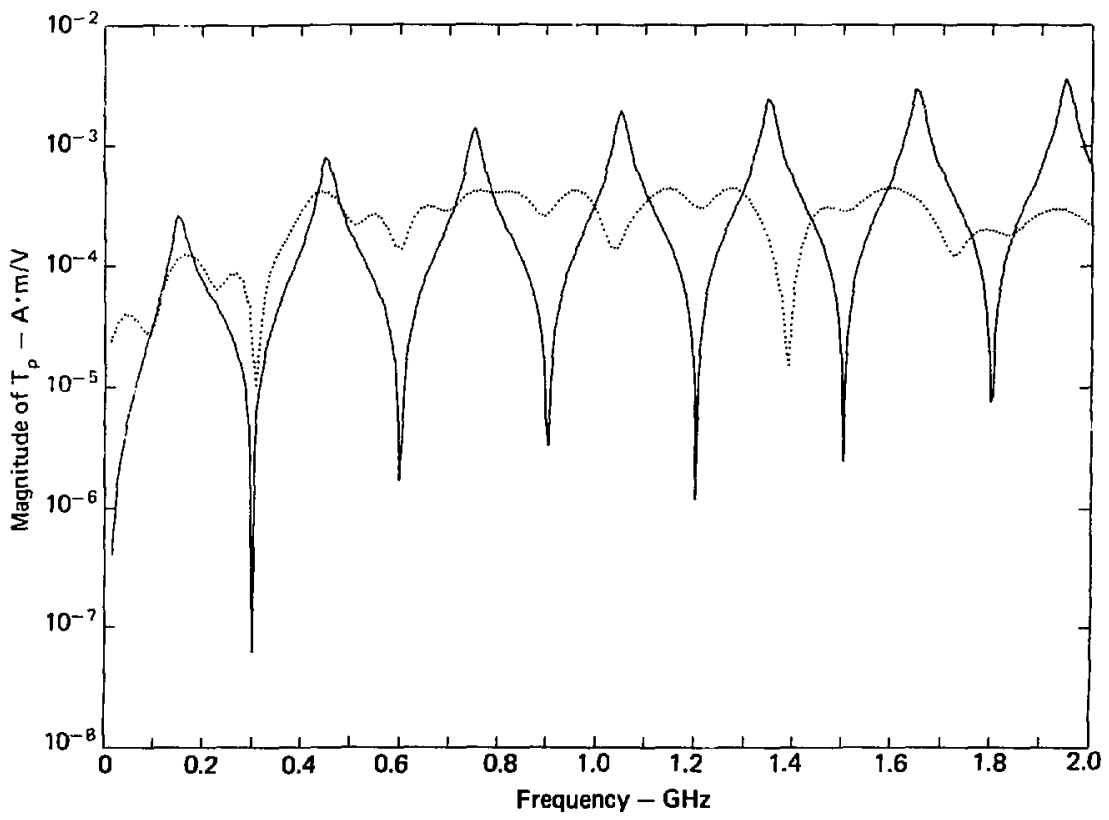

Plot 14. Magnitude of the trausfer function of the current at the base of a wire behind a plane with aperture from an iacident plane wave, $T_{\text {. }}$. In this plot, the aperture is a circle with $\mathbf{~} 0.16-\mathrm{m}$ diameter. The wire is $0.05 \mathrm{~m}$ behind the plane. The solid and dotted curves represent the mimerical and experimental results, respectively. 


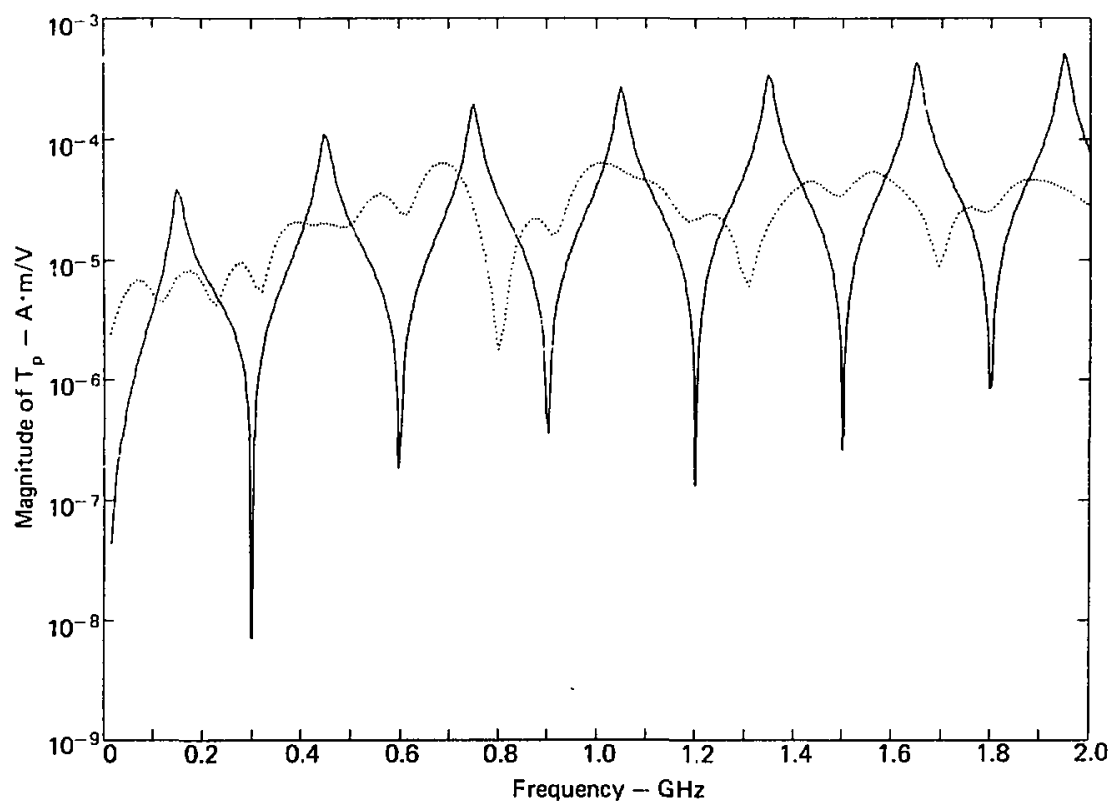

Plot 15. Same graph as Plot 14, except the aperture is a circle with $\mathbf{0 . 1 6 - m}$ diameter. The wire is $0.35 \mathrm{~m}$ behind the plane. 


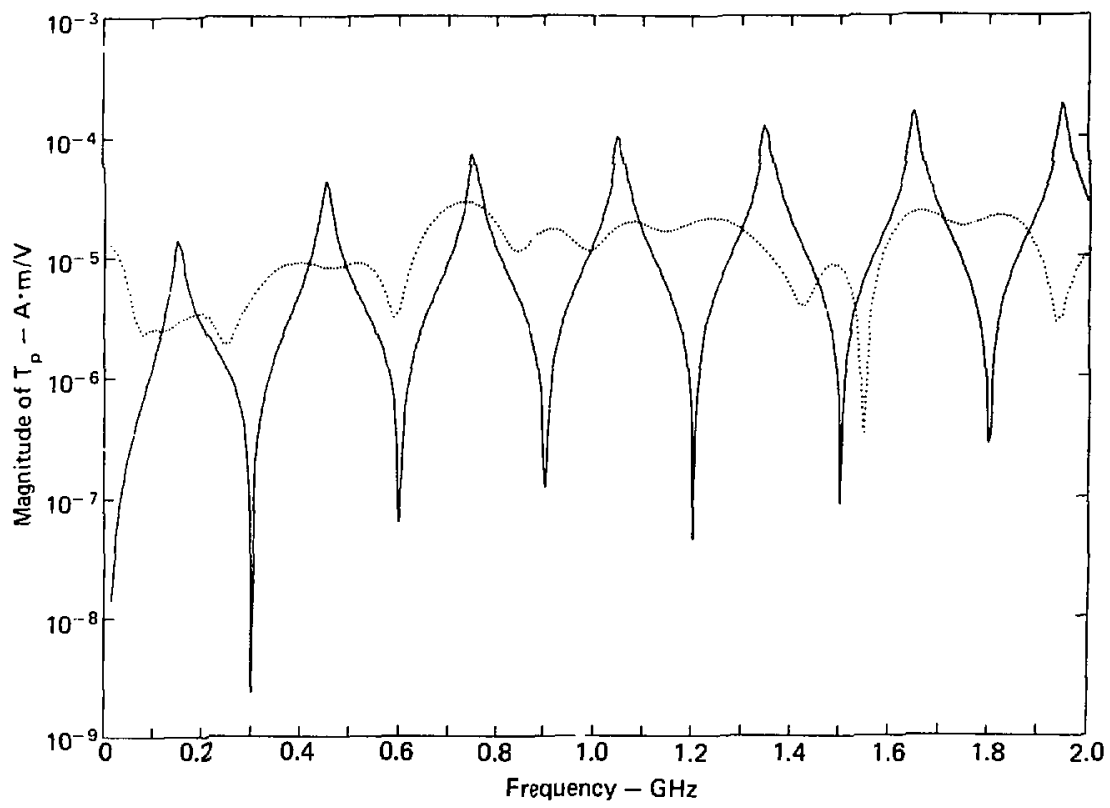

Plot 16. Same graph as Plot 14, except the aperture is a cirile with a 0.16 -m diameter. The wire is $0.95 \mathrm{~m}$ behind the plane. 


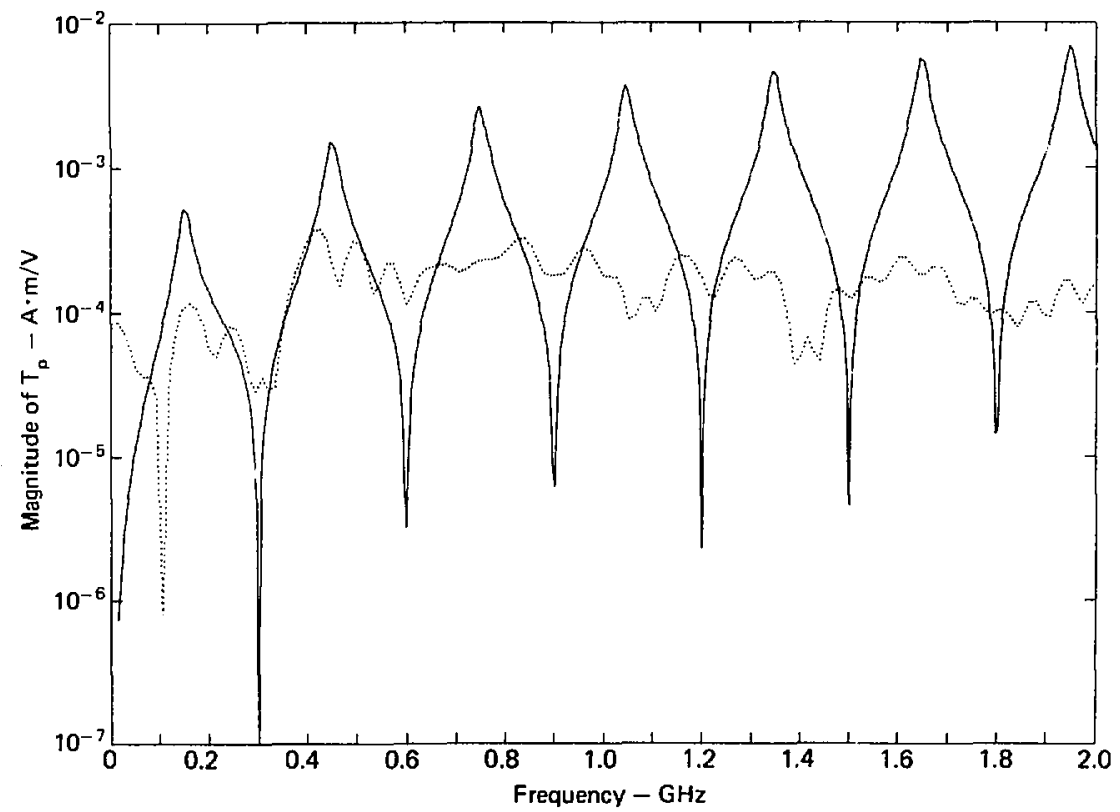

Plot 17. Same graph a.s Plot 14, excep! the aperture is a circle with a $0.02-m$ diameter. The wire is $0.05 \mathrm{~m}$ behind the plane. 


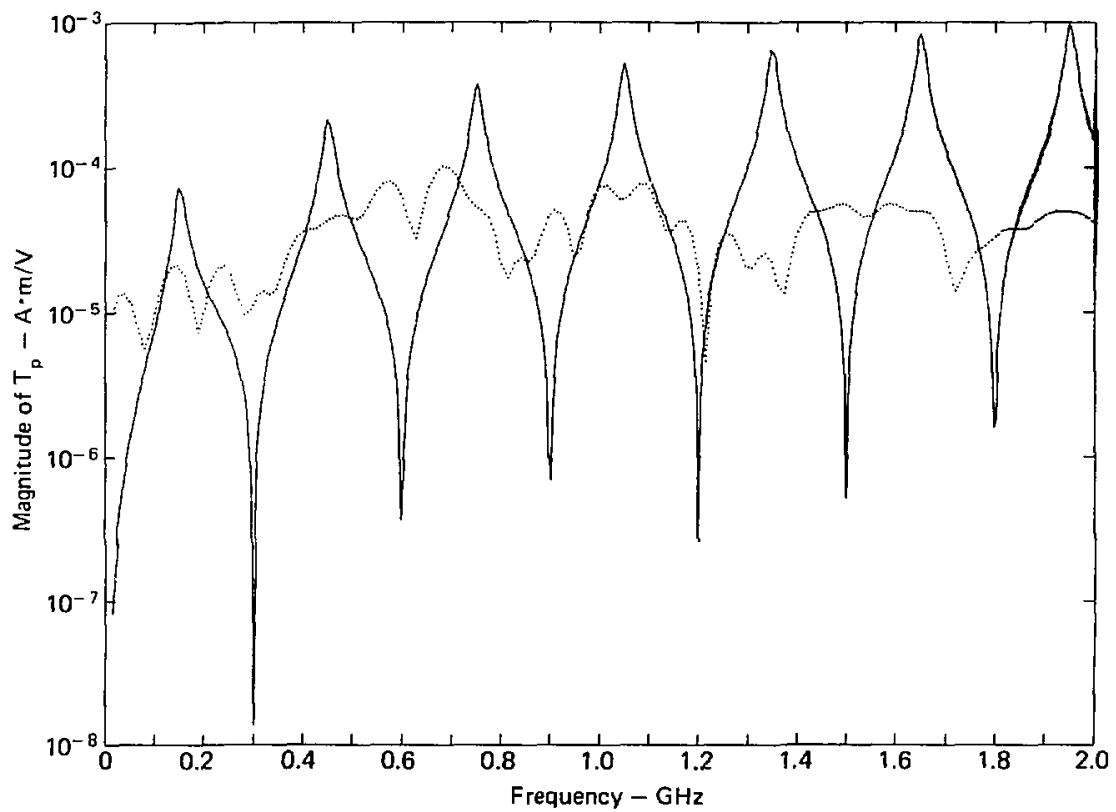

Plot t8. Same graph as Plot 14, except the aperure is a circle with a $0.2-\mathrm{m}$ diameter. The wire is $0.35 \mathrm{~m}$ behind the plane. 


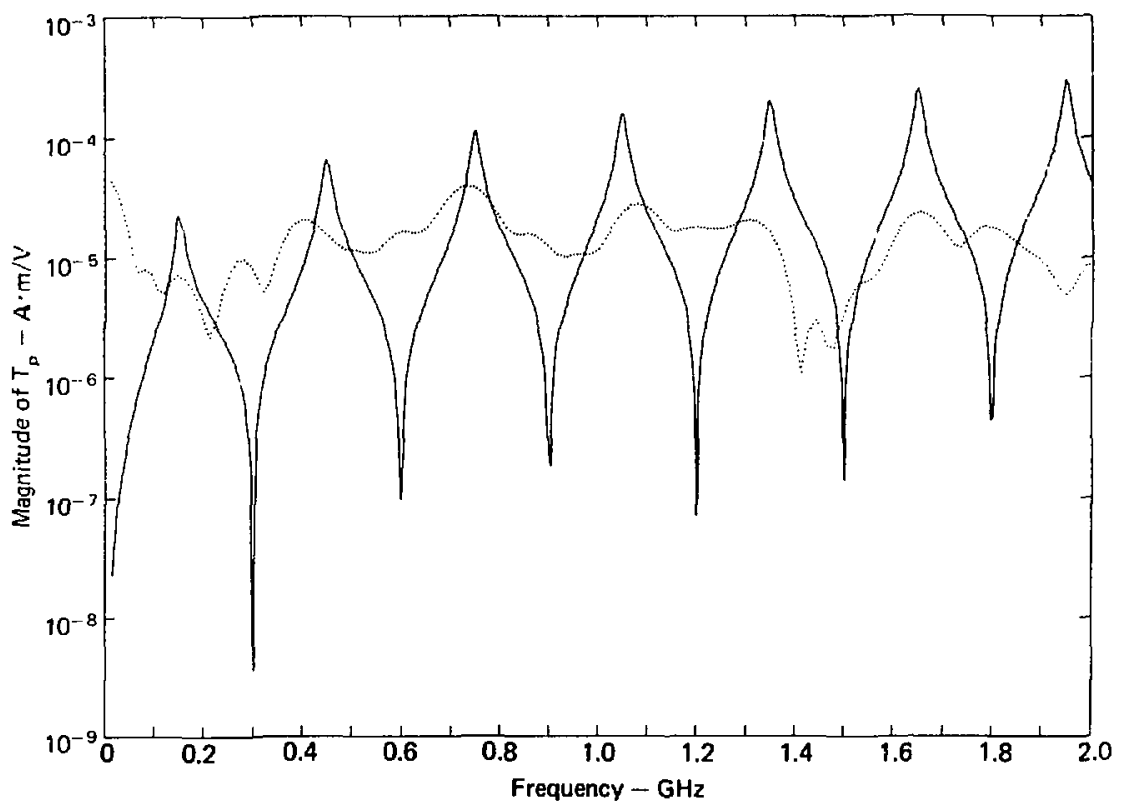

Plot 19. Same graph as Plet 14, except the aperture is a circle with a $0.2-m$ dimmeter. The wire is $1.15 \mathrm{~m}$ behind the plane. 


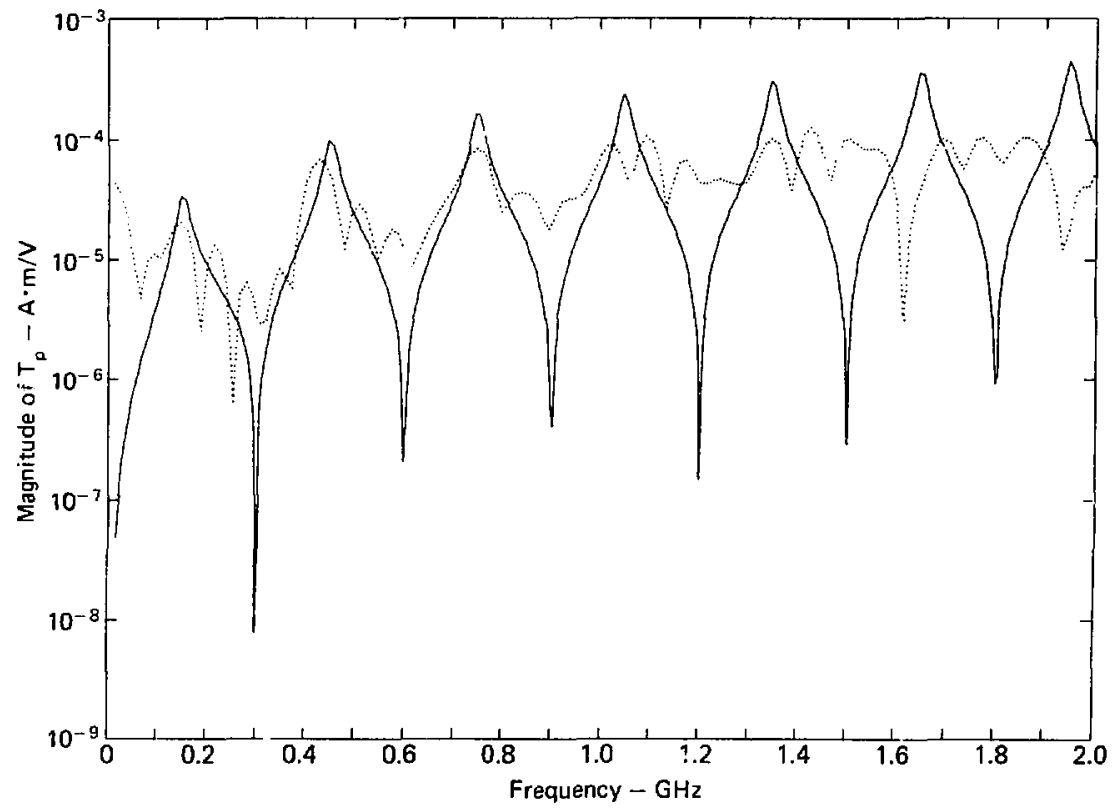

Plo1 20. Same graph as Plot 14, except aperture height and width ase $0.08 \mathrm{~m}$. The wire is $0.05 \mathrm{~m}$ behind the plane. 


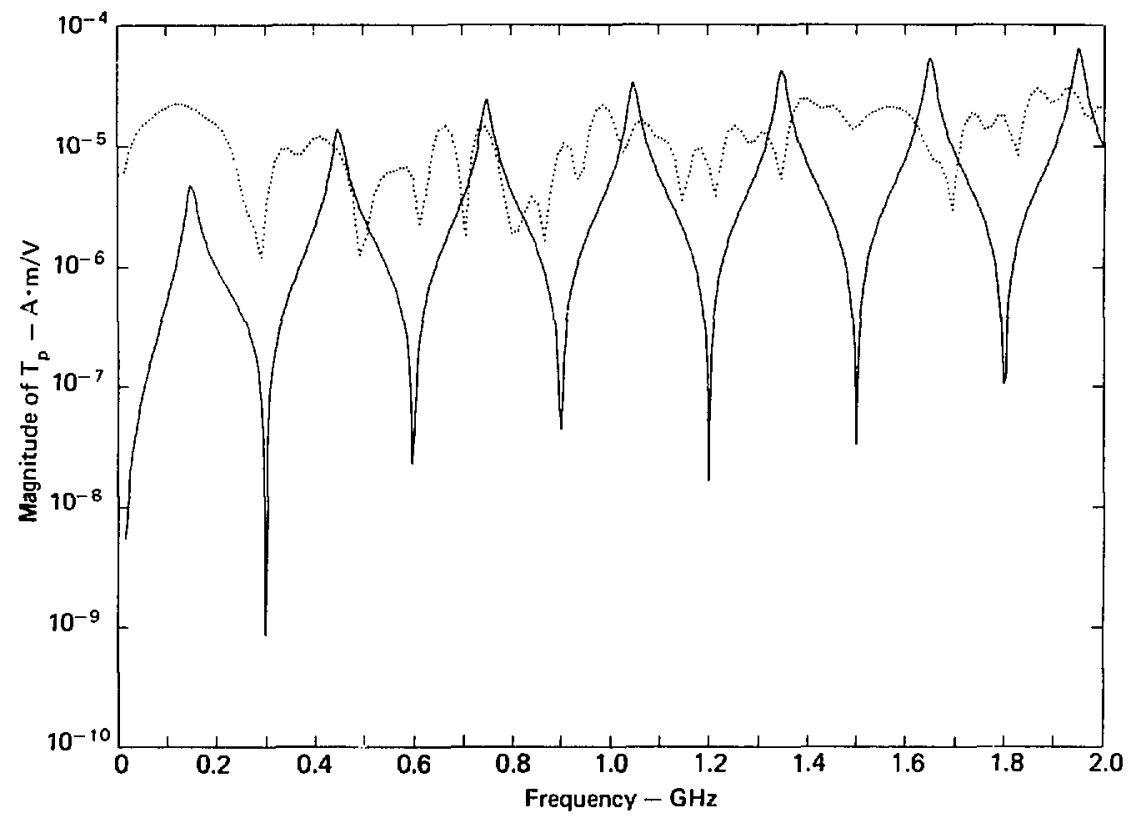

Plo1 21. Same graph as Plot 14, except aperture height and width are $0.08 \mathrm{~m}$. The wire is $0.35 \mathrm{~m}$ behind the plane. Note that the experimental data given here are incorrect. 


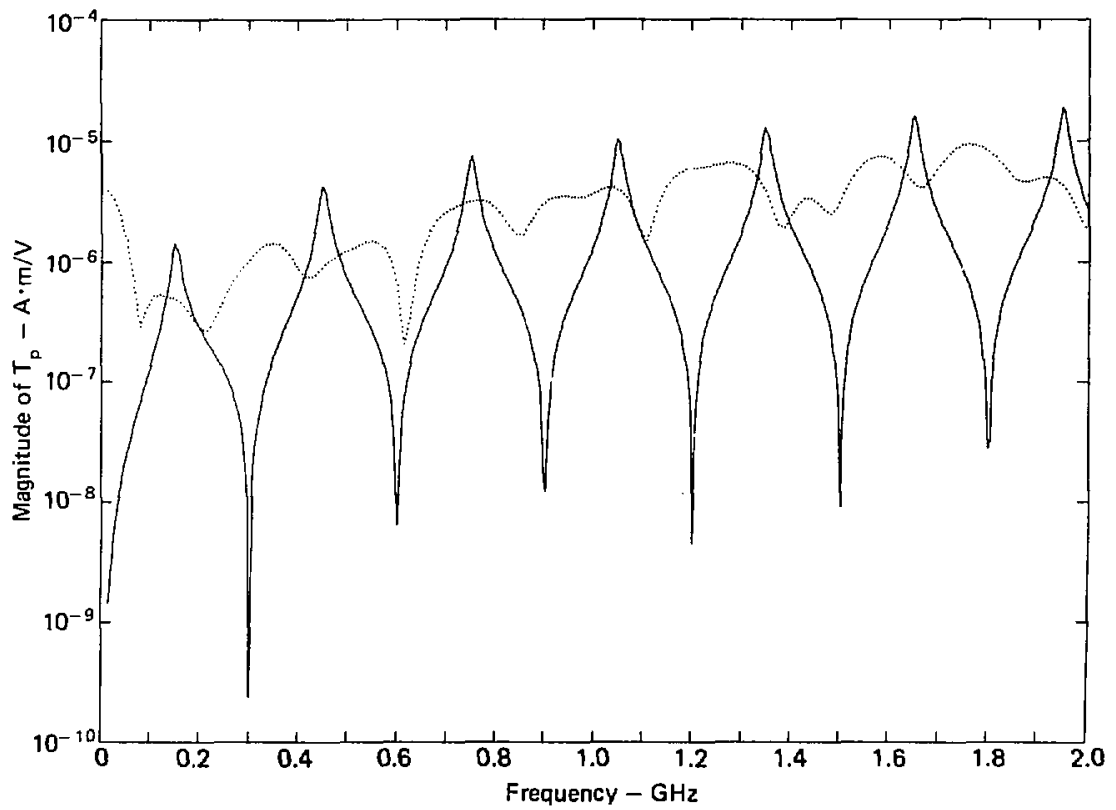

Plot 22. Same graph as Plot 14, except aperture height and width are $0.08 \mathrm{~m}$. The wire is $1.15 \mathrm{~m}$ behind the plane. 


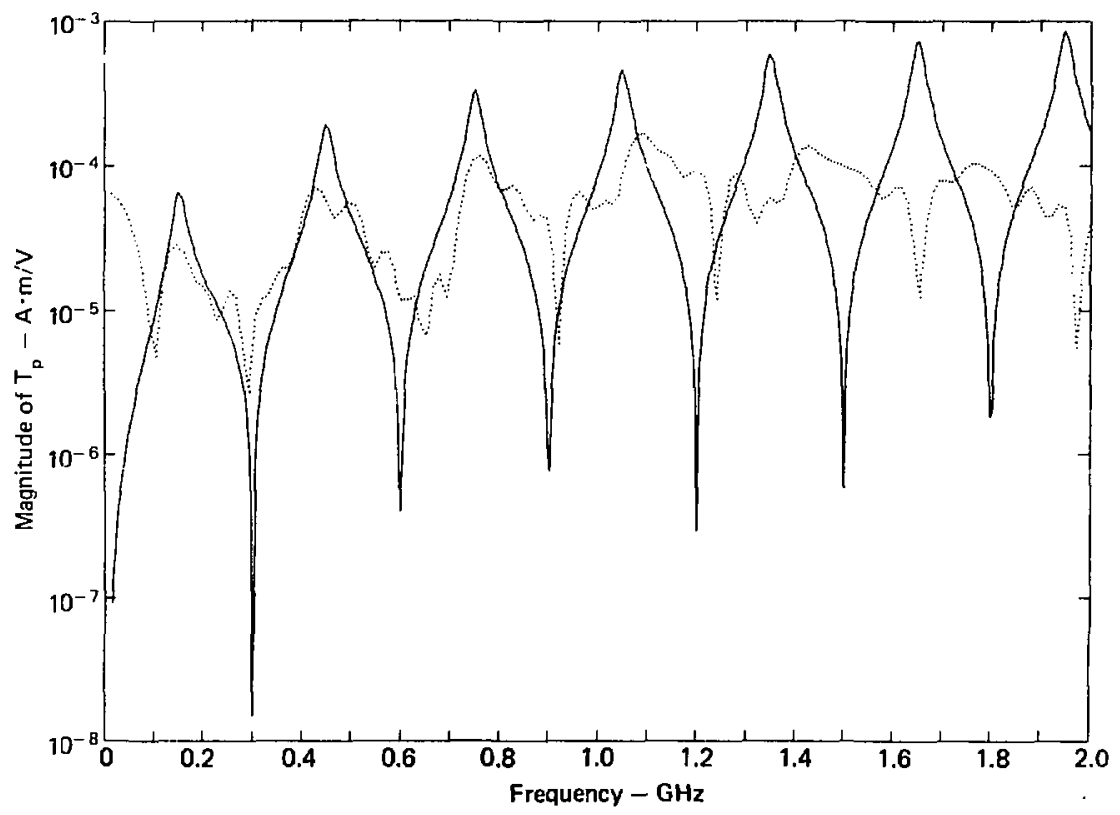

Plot 23. Same graph as Plot 14, except aperture height and width are $0.1 \mathrm{~m}$. The wire is $0.05 \mathrm{~m}$ behind the plane. 


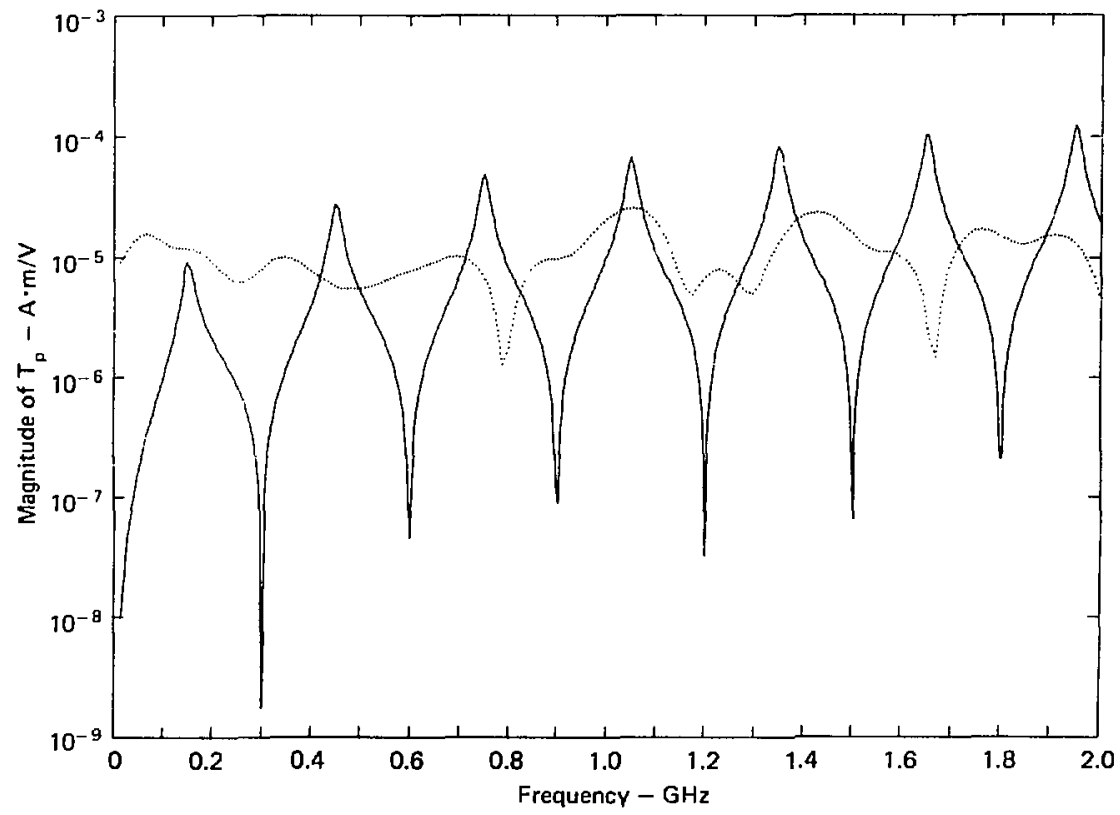

Plot 24. Same gnaph as Plot 14, except aperture helght and width are $0.1 \mathrm{~m}$. The wire is $0.35 \mathrm{~m}$ behind the plane. Note that the experimental dats given here ure incorrect. 


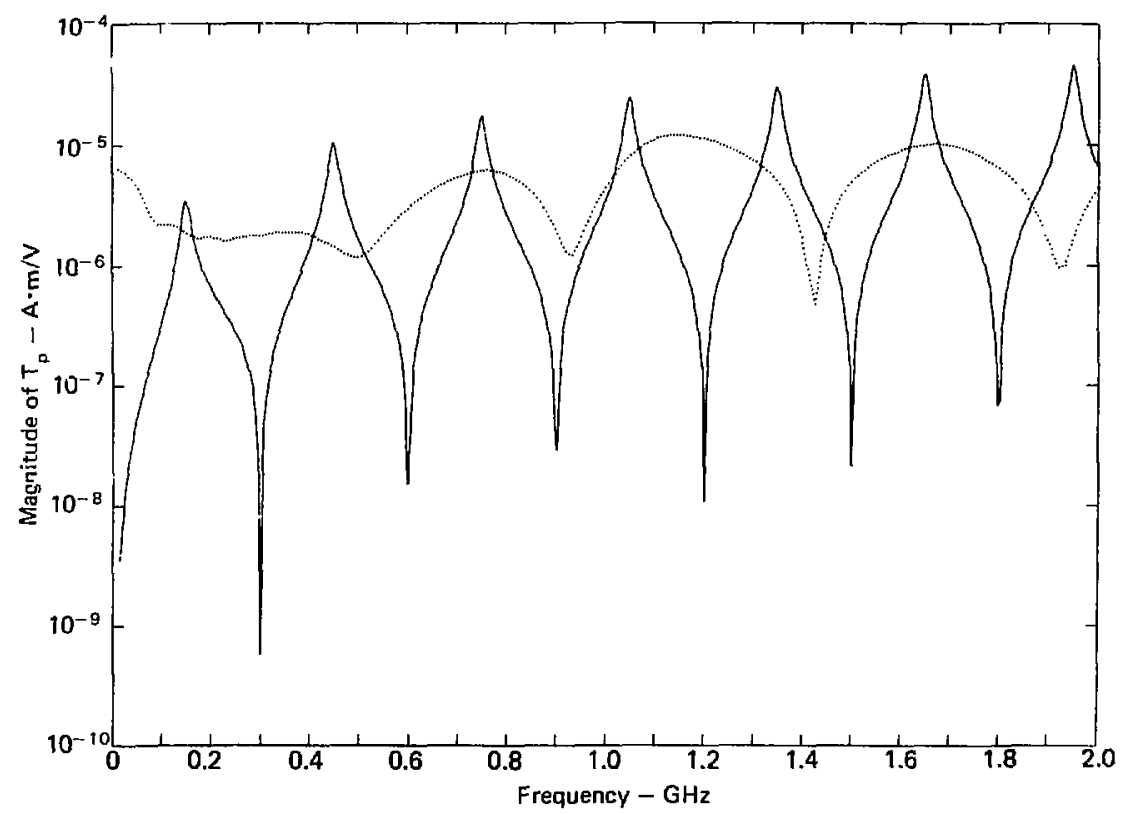

Pot 25. Same graph as Plot 14, except aperture height and width are $0.1 \mathrm{~m}$. The wire is $0.95 \mathrm{~m}$ behind the plane. 


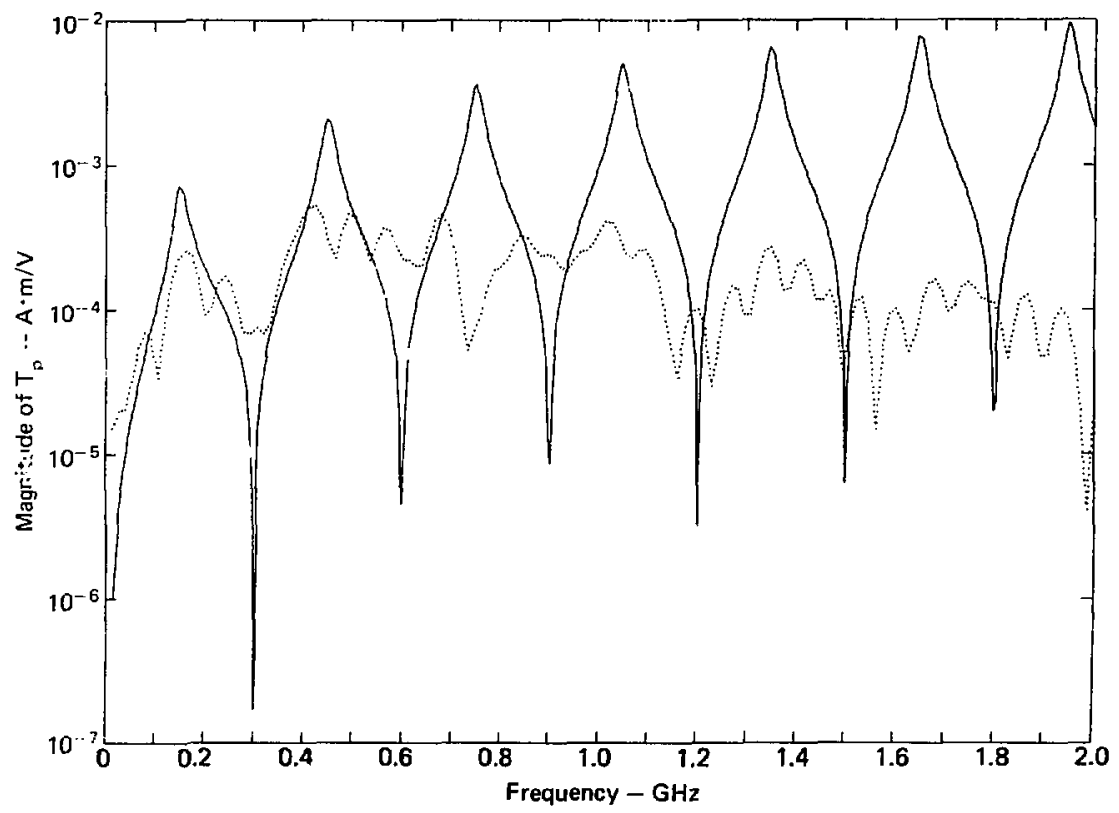

Flot 26. Same graph as Plox 14, except aperture height and width are 0.3 and $0.2 \mathrm{~m}$, respectively. The wire is $0.05 \mathrm{~m}$ behind tbe planc. 


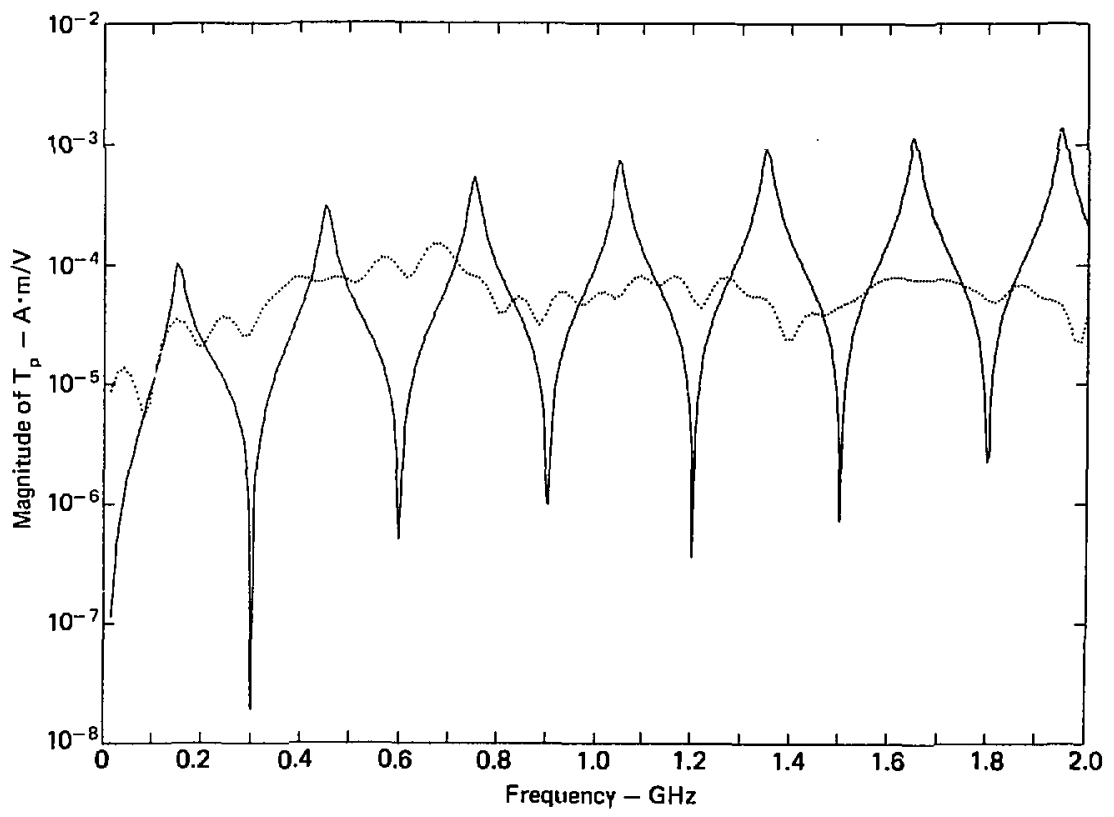

Plot 27. Same graph es Plot 14, except aperture height and width are 0.3 and $0.2 \mathrm{~m}$, respectively. The wire is $0.35 \mathrm{~m}$ tehind the plane. 


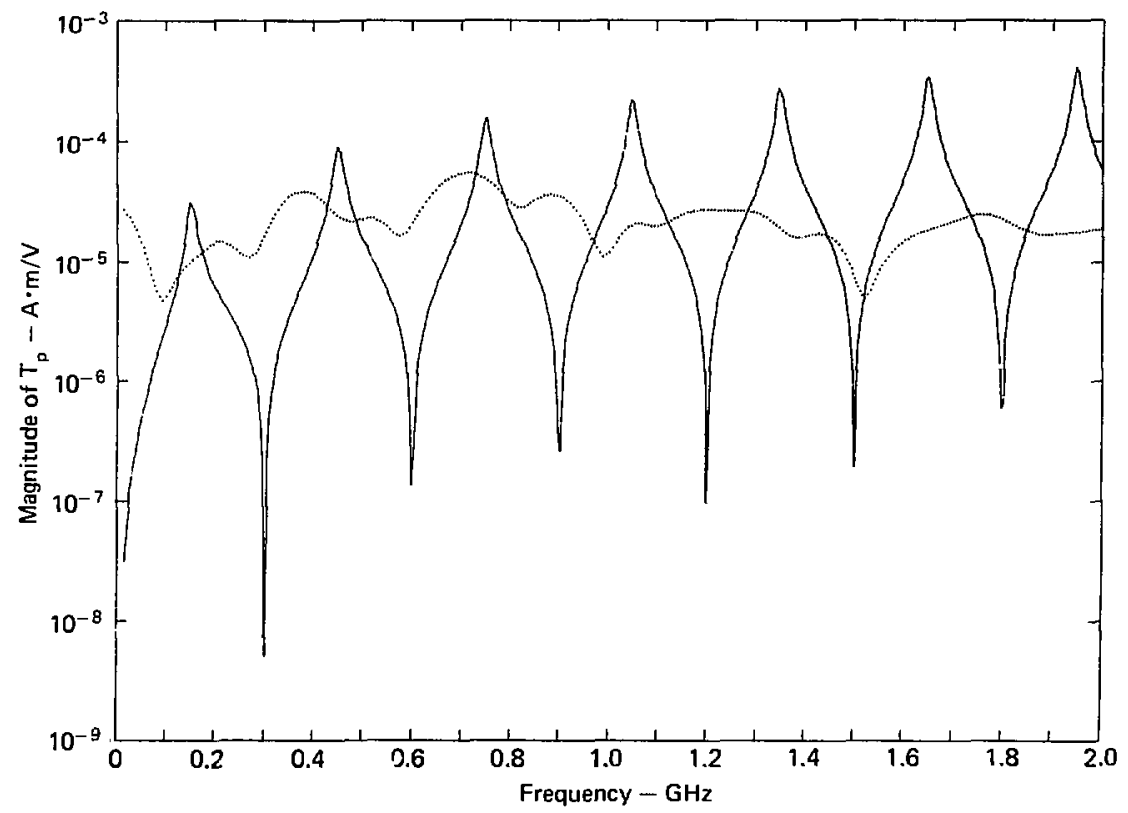

Plot 28. Same graph as Plot 14, except aperture height and width are 0.3 and $0.2 \mathrm{~m}$, respectively. The wire is $1.15 \mathrm{~m}$ behind the plane. 
the thin cylinder. The first transfer function, the normalized current density on the outside surface of the cylinder, is calculated by a very different procedure for the fat cylinder than was used for the thin cylinder. For the fal cylinder, the patch capability of $\mathrm{NEC}^{4}$ is used to solve for the surface current on the outside of the cylinder. The total transfer function for the fat cylinder is then calculated by using Eq. (16).

Plots 29-31 show the frequency plot of the normalized magnitude of the surface current density at the point where the aperture will be placed in the outside surface of the fat cylinder. Cases where the incident plane wave is head on, $90^{\circ}$ from head-on, and backside of the cylinder are presented. Table 5 (page 40) presents the values of the Bethe hole coejficients $T_{2}$ for the apertures cut into the fat cylinder; [these apertures are presented in Table 3 (page 9)]. Plot 32 shows the frequency plot of the normalized magnitude of current $T_{3}$ at the base of the wire from a magnetic dipole source on the inside surface of the cylinder. Plots 33.44 display the frequency plot of the normalized magnitude of the current $T_{T}$ at the base of the wire from an incident plane wave. These plots present both the numerical and experimental results; the solid and dotted curves represent the numerical and experimental data, respectively. These plots show the data for the five aperture and three different arrival angles of the incident plane wave. (Text continues on page 57.)

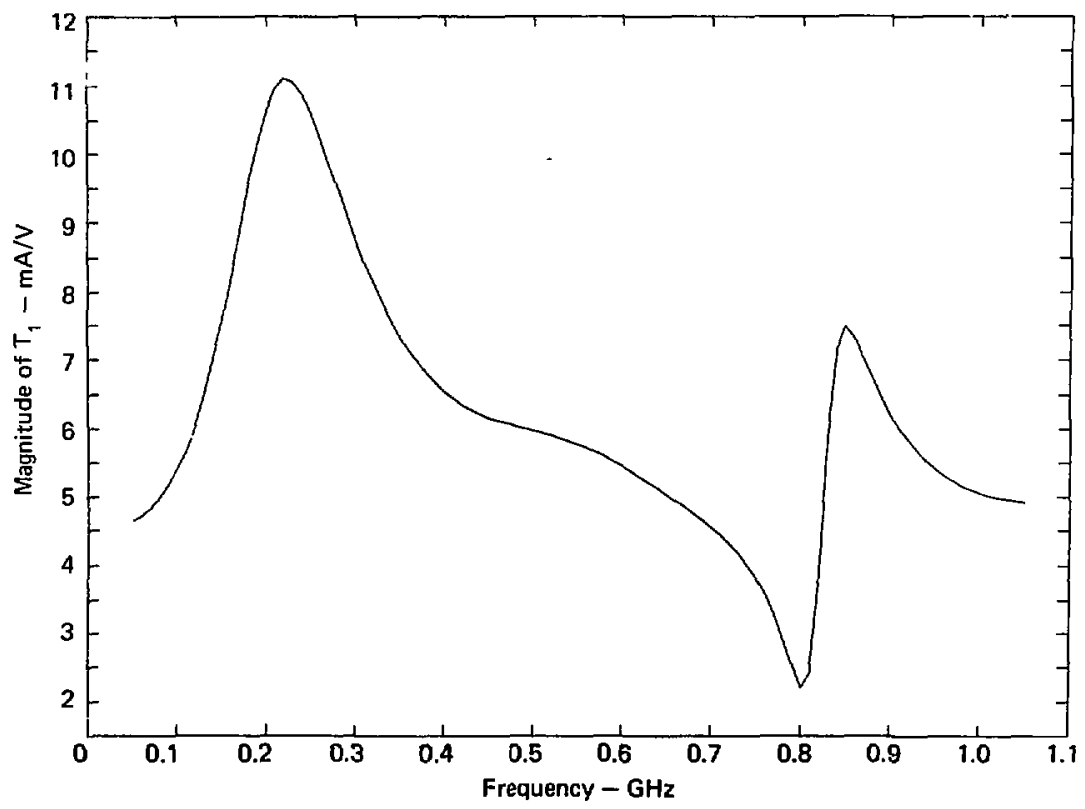

Plot 29. Mugnitude of the transfer function of the external current density at the center of an electrically lnrge cylinder from an incident plane wave, $T_{T}$ The results were muerically evaluated by Eq. (15). The polnt showa here is on the illuminated side of the cylinder. 


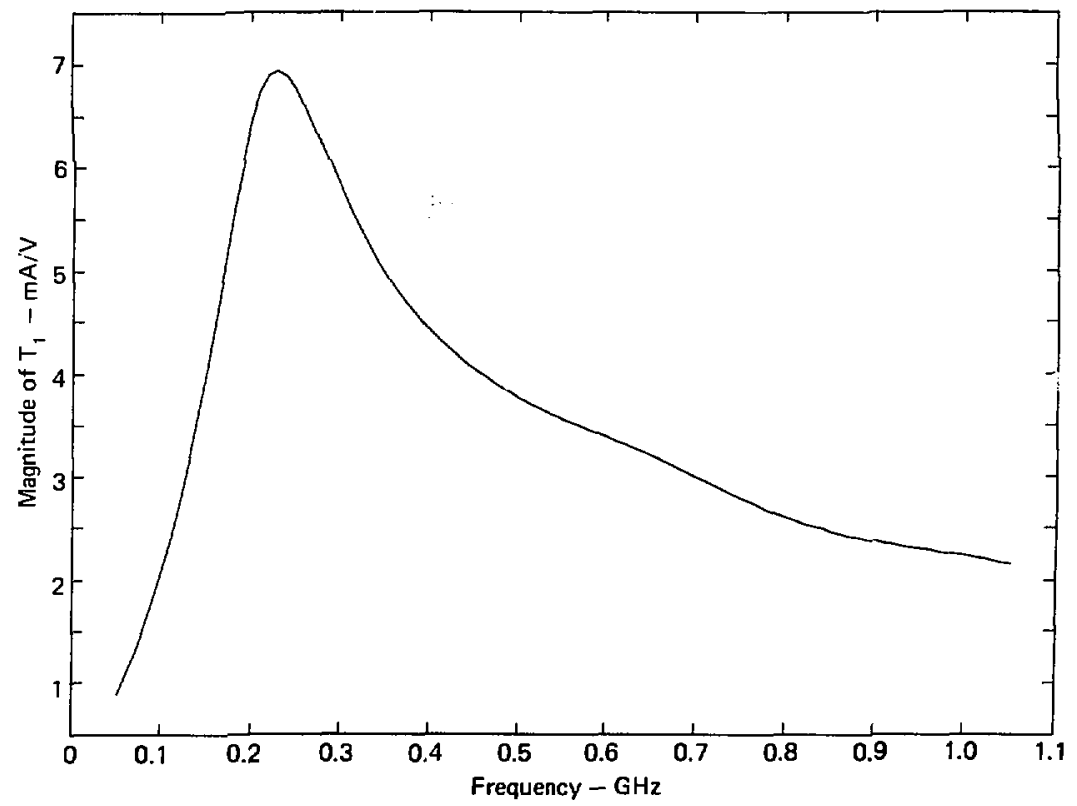

Plot 30. Same graph as Plot 29, except the point on the cylinder is $90^{\circ}$ to head-on incidence. 


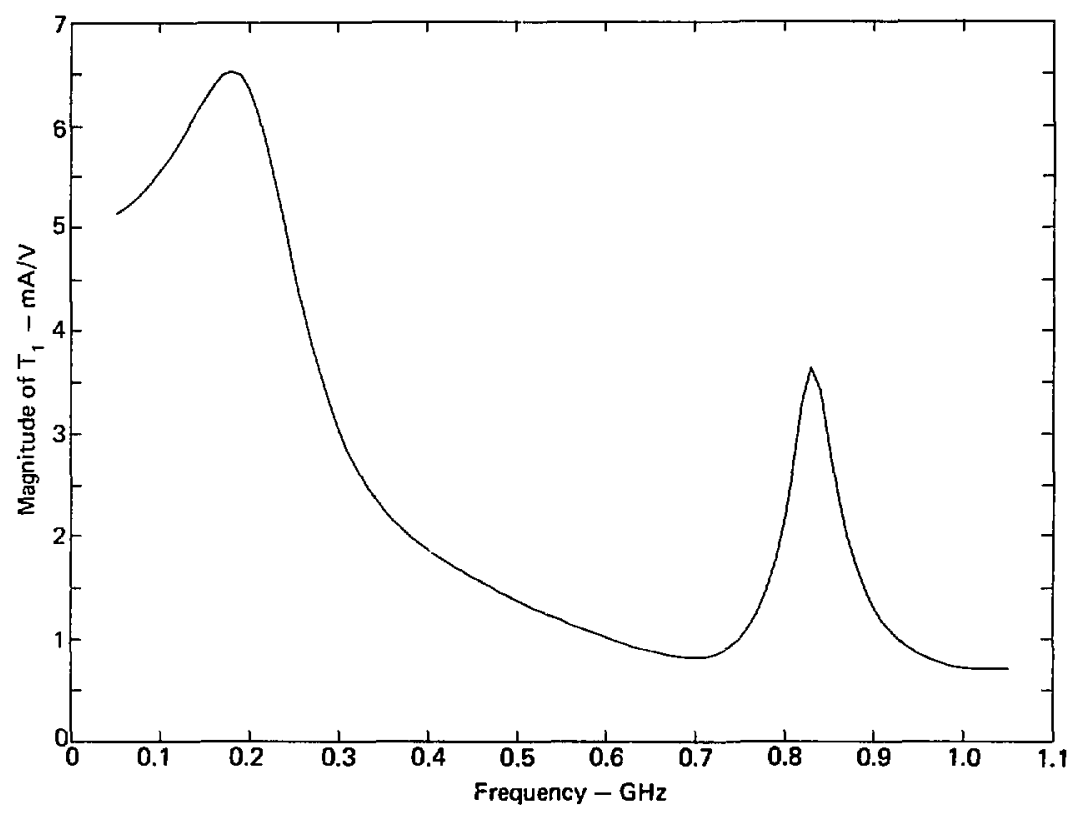

Plot 31. Same graph as Plot 29, except the point is on the shaded side of the cylinder.

:.*

Table 5. Values of Bethe hole coefficient for apertures in fat cylinder experimer:s. ${ }^{2}$

\begin{tabular}{clc}
\hline & \multicolumn{2}{c}{$\begin{array}{c}\text { Dimenslons, } \\
\text { m }\end{array}$} \\
\hline 1 & 0.10 (circular) & $-1.665 \times 10^{-4}$ \\
2 & $0.15 \times 0.10$ & $-2.293 \times 10^{-4}$ \\
3 & $0.10 \times 0.15$ & $-4.216 \times 10^{-4}$ \\
4 & $0.30 \times 0.05$ & $-1.006 \times 10^{-4}$ \\
3 & $0.10 \times 0.20$ & $-8.307 \times 10^{-4}$ \\
\hline
\end{tabular}

2These results were numerically evaluated from Eqs. (5b) and (5c). 


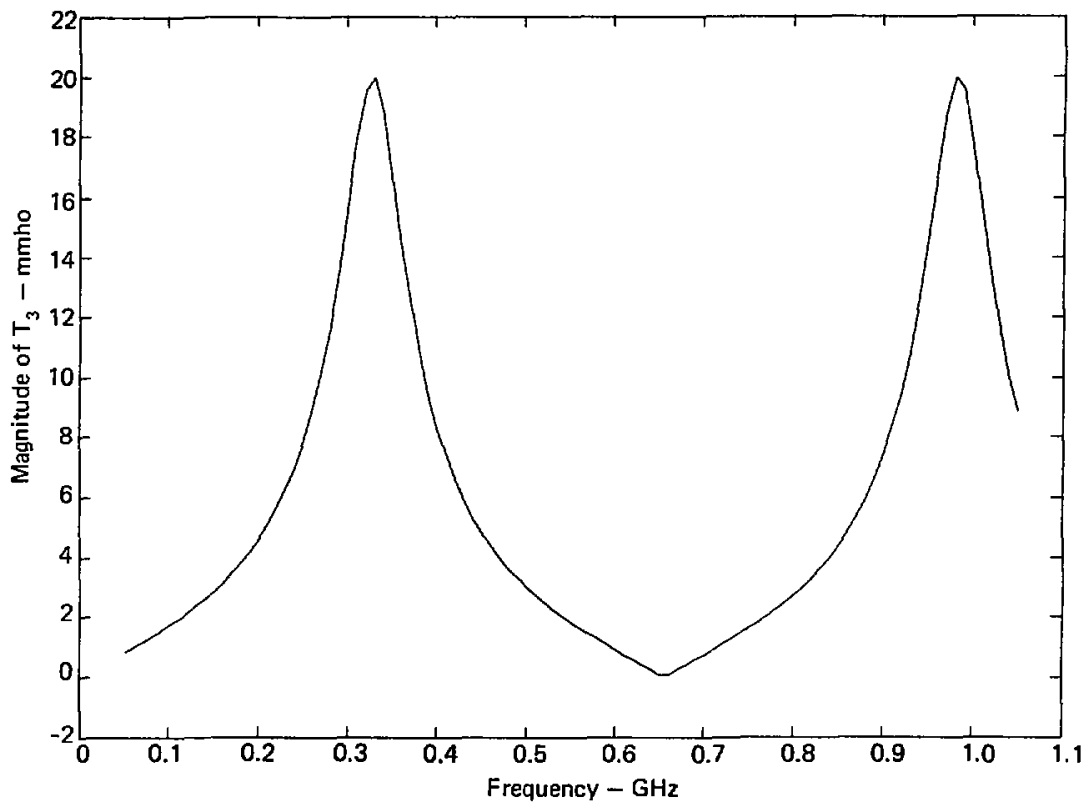

Plot 32. Magnitude of the tmansfer function of the current at the base of the vire from a magnetic dipole source on the surface of the fal cylin. der, $T_{3}$. These results were numerically evaluated from the term inside the brackets in $\mathbf{L}_{4}$. (7a). 


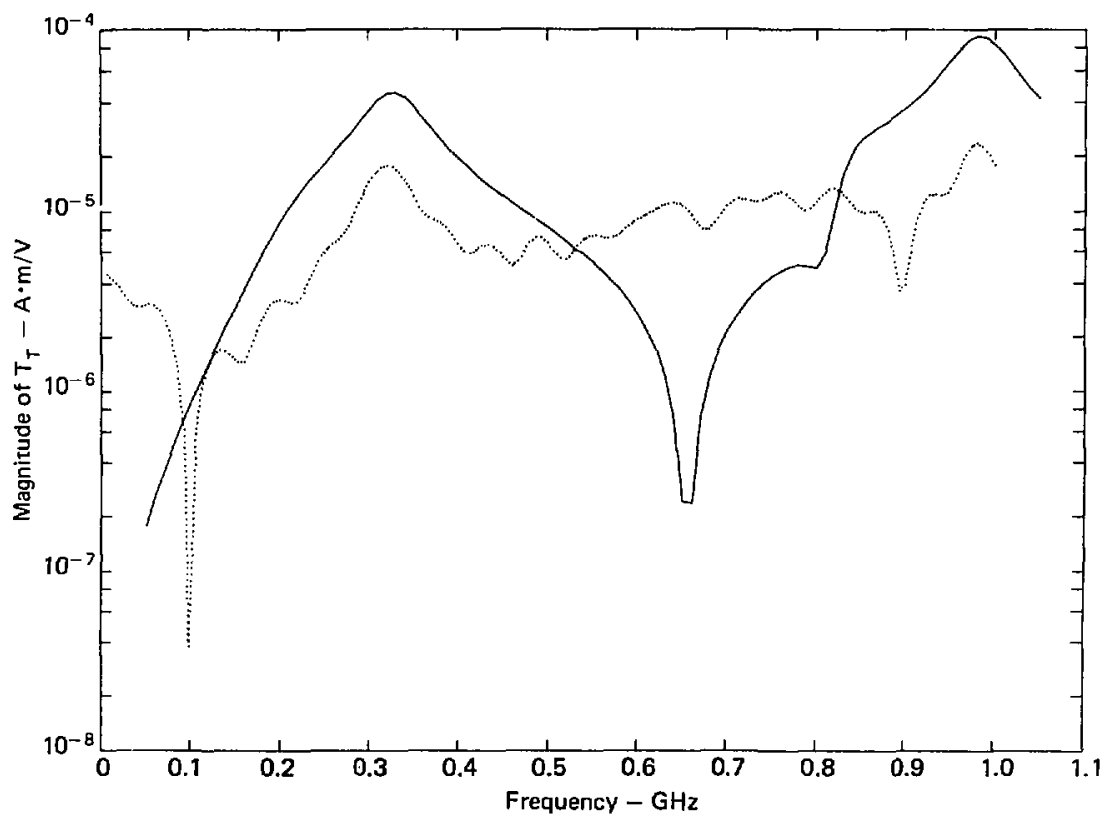

Plot 33. Magnitude of the transfer fuaction of the current at the base $n$ f the wire from an incident plane wave on the fat cylinder, $T_{T}$. In this plot the aperture is a circle with $a 1-m$ diametcr. The aperture is on the illuminated side of the cylinder. The solid and dotted curves represent numerical und experimental results, respectively. 


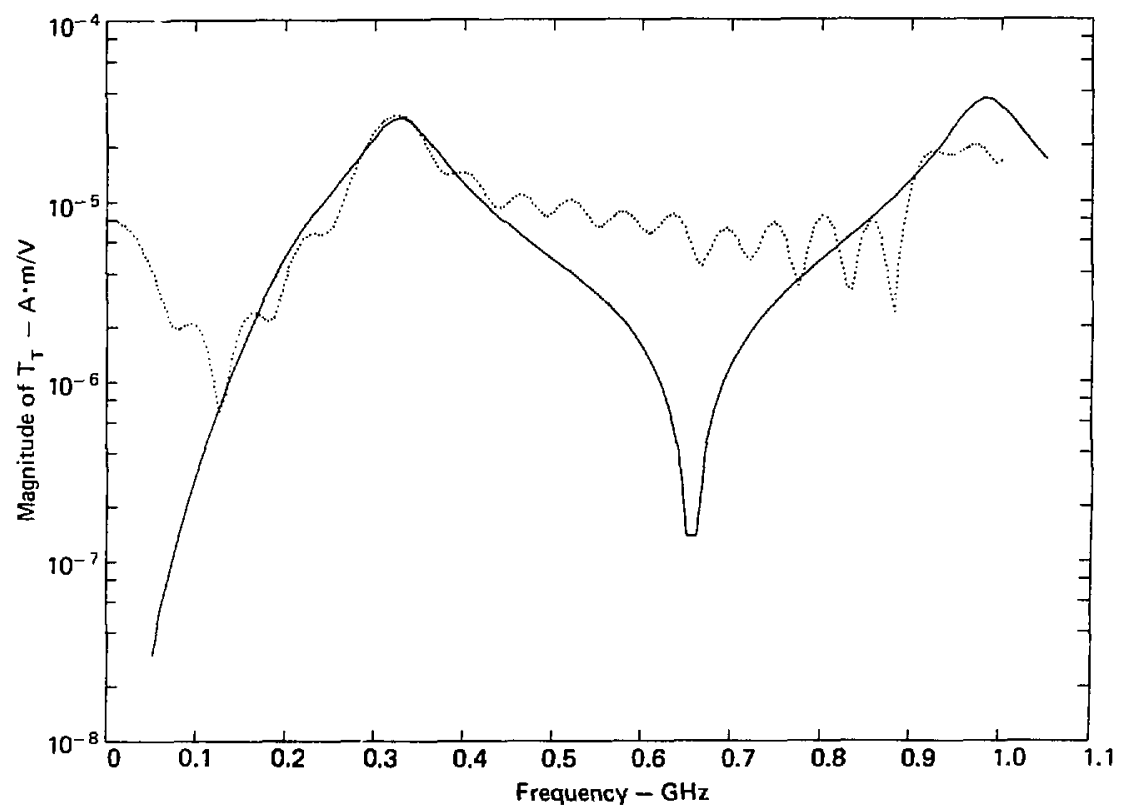

Plot 34. Same graph as Plot 33, except the aperture is a circle with a $0.1-m$ diameter. The aperture is on the side of the cylinder that is $90^{\circ}$ from hegd-on incidence. 


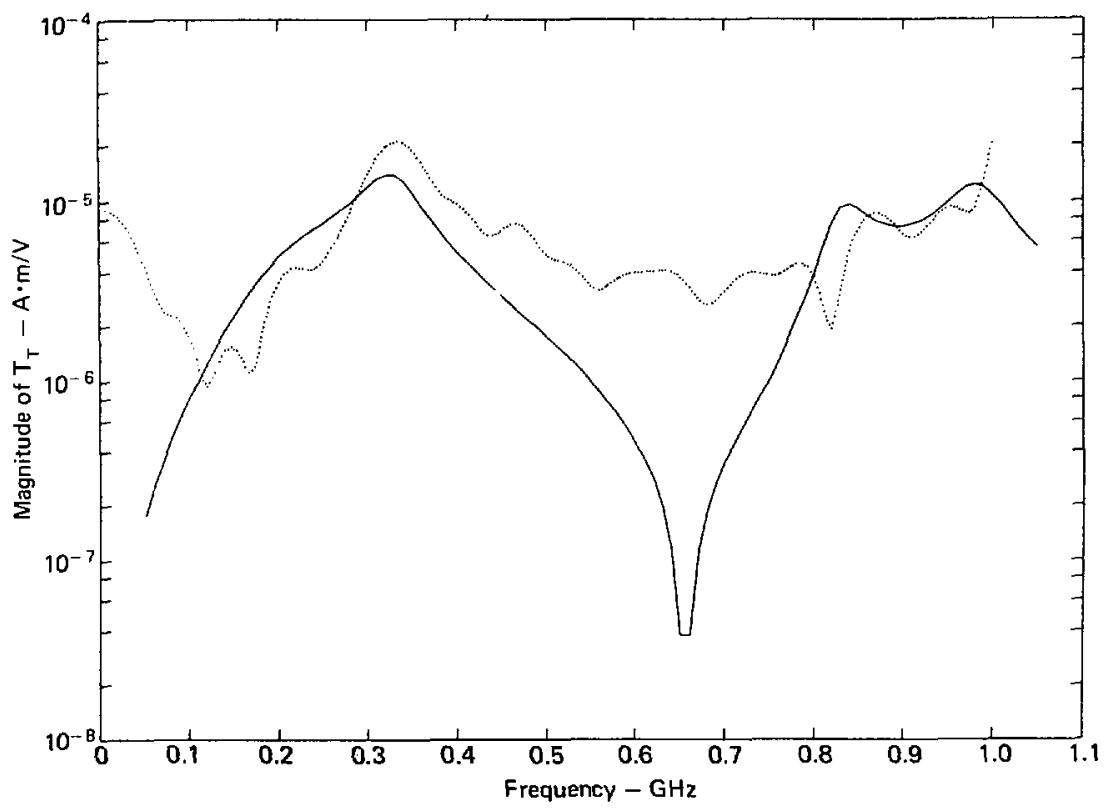

Plot 35. Same graph as Plot 33, except the apertures is a circle with a $0.1-m$ diameter. The aperture is on the shaded side at the cylinder. 


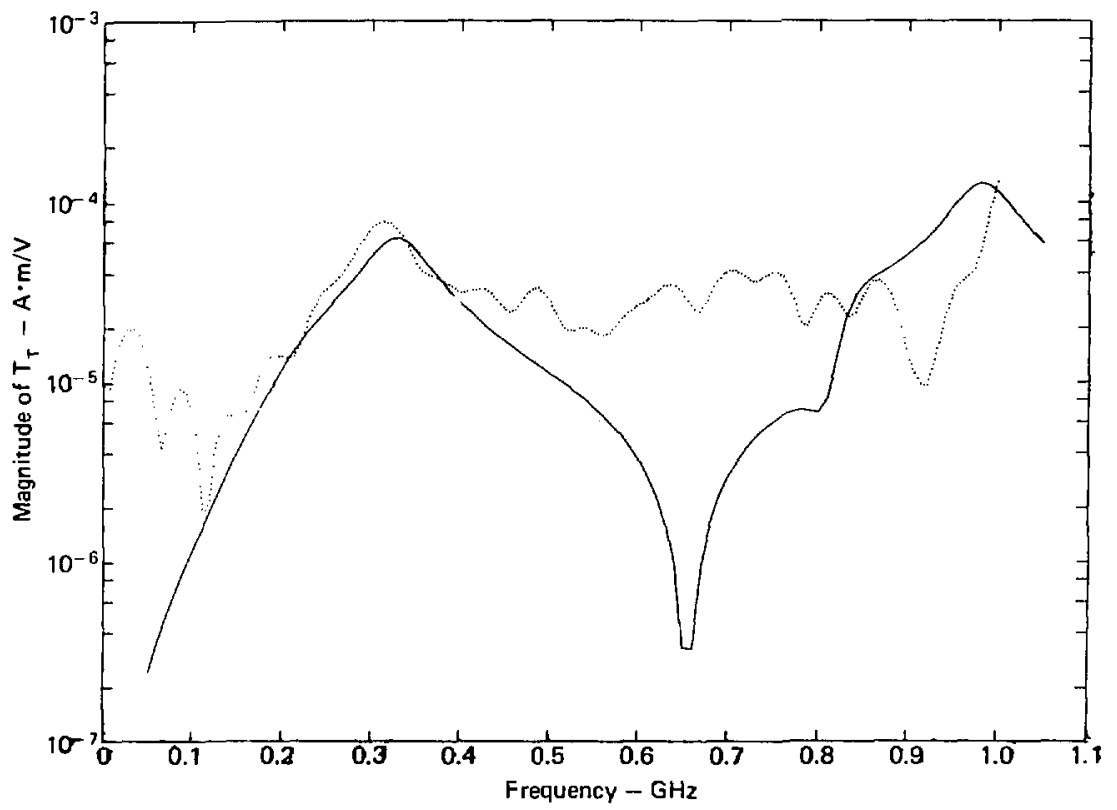

Phot 36. Sam graph ay : lot 33, except the wxib and circunferential dimensions of the aperture are 0.15 and 0.1 m, respectlvely. The apernere is on the illomineted side of the cylinder. 


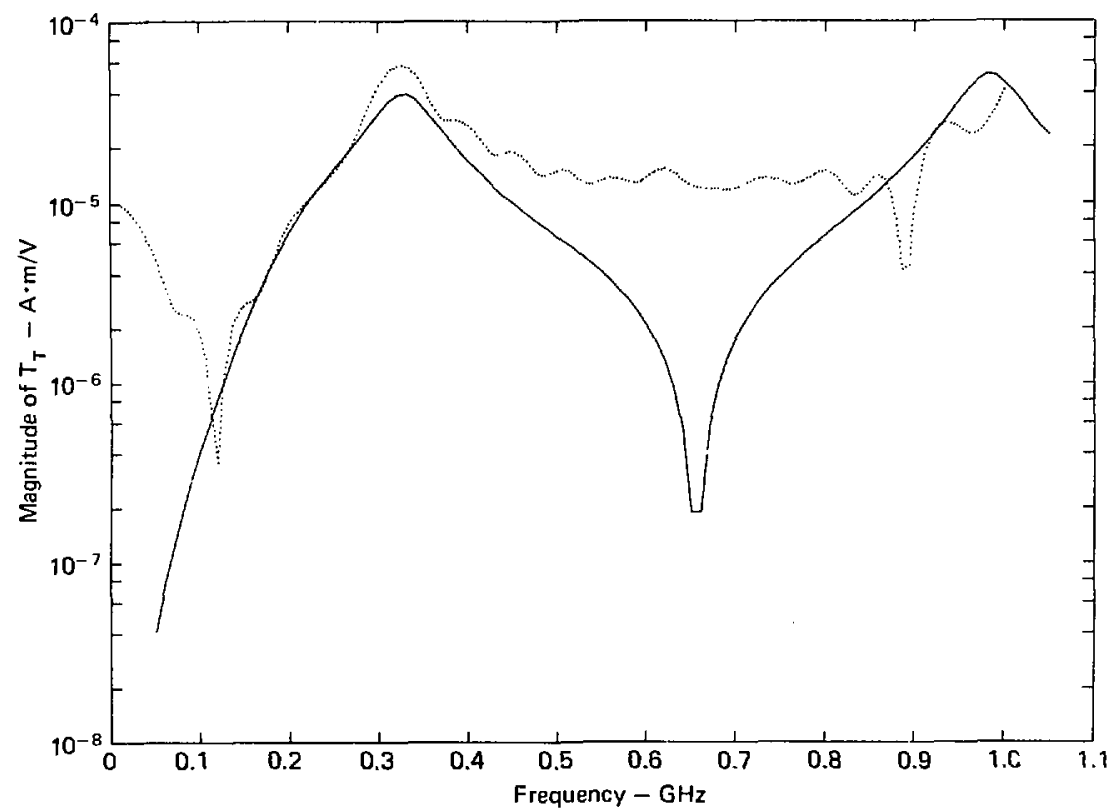

Plot 37. Same graph as Piot 33, except the axial and circumferential dimensions of the aperture are 0.15 and 0.1 m, respectively. The aperture is on the side of the cylinder that is $90^{\circ}$ Irom head-on incidence. 


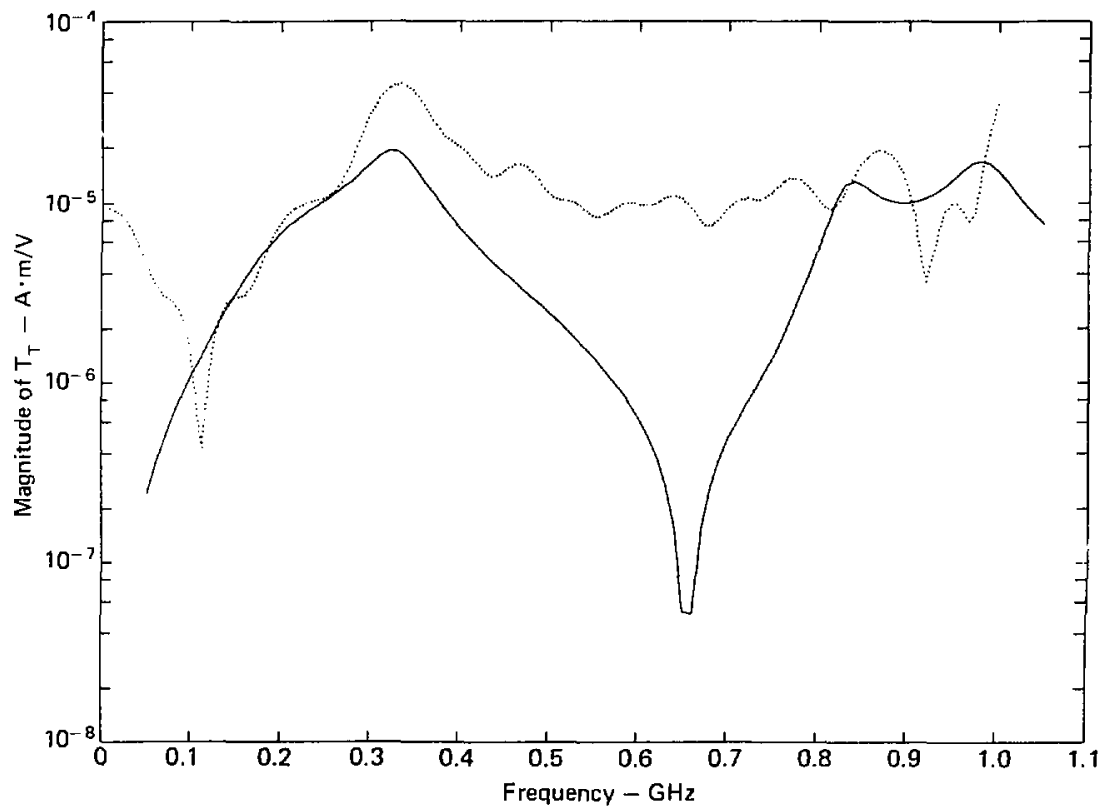

Plot 38. Same graph as Plot 33, except the axial and circumferential dimensions of the aperture are $0.1 f$ and $0.1 \mathrm{~m}$, respectively. The aperture is on the shaded side of the cylinder. 


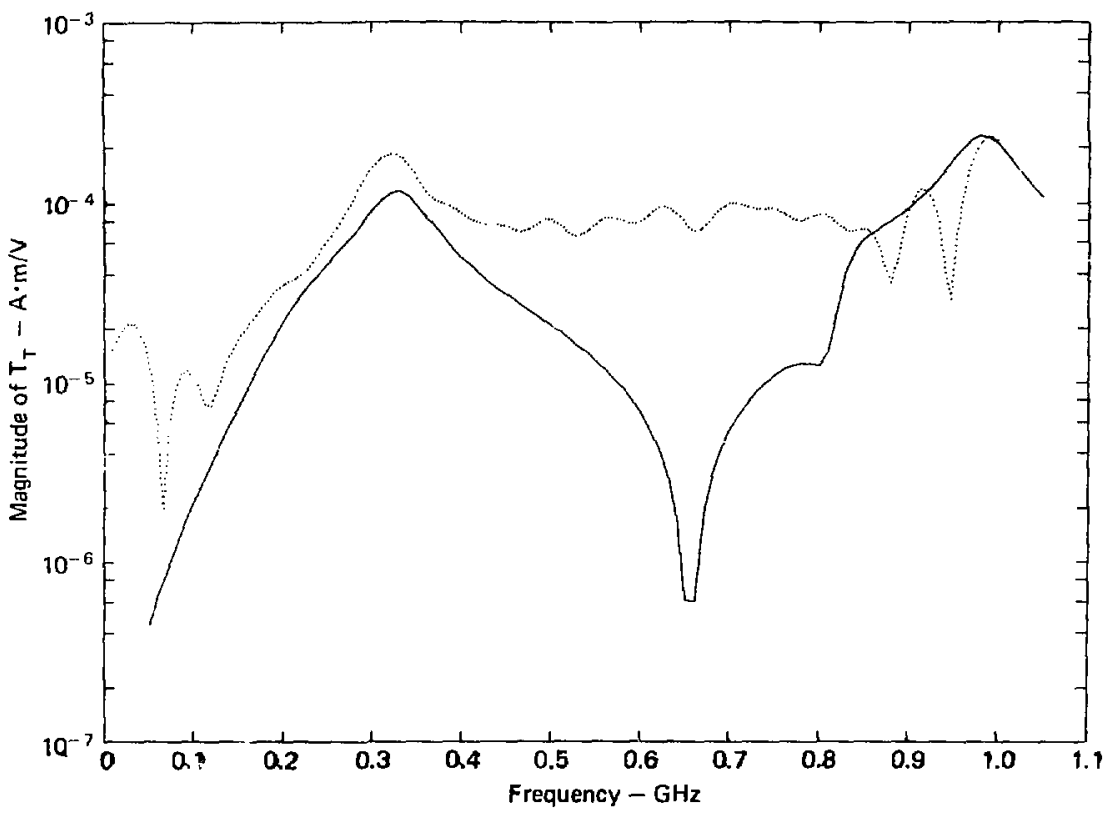

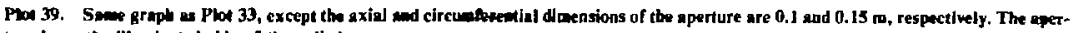
ture is on the illuminated side of the eylinder. 


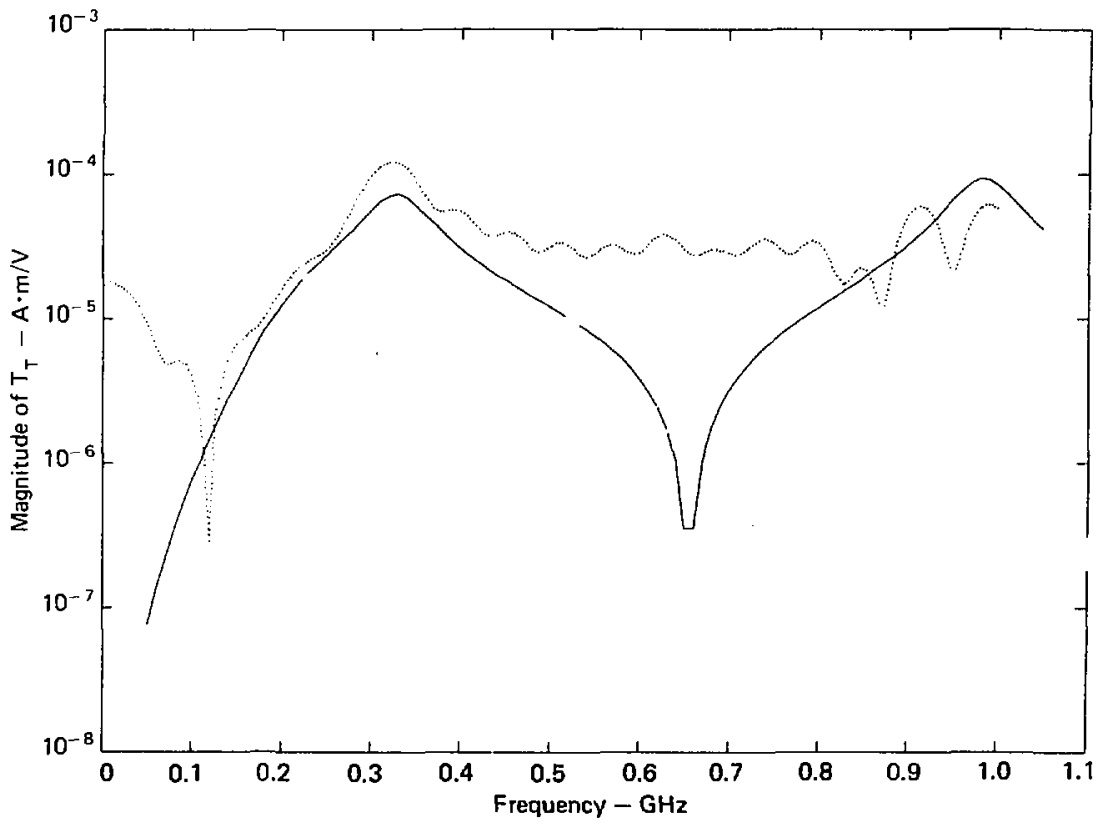

Plot 40. Same graph as Plot 33, except the axial and circumferential dimensions of the apert ure are 0.1 and $0.15 \mathrm{~m}$, respectively. The aperture is on the side of the cylinder that is $90^{\circ}$ from head-on incidence. 


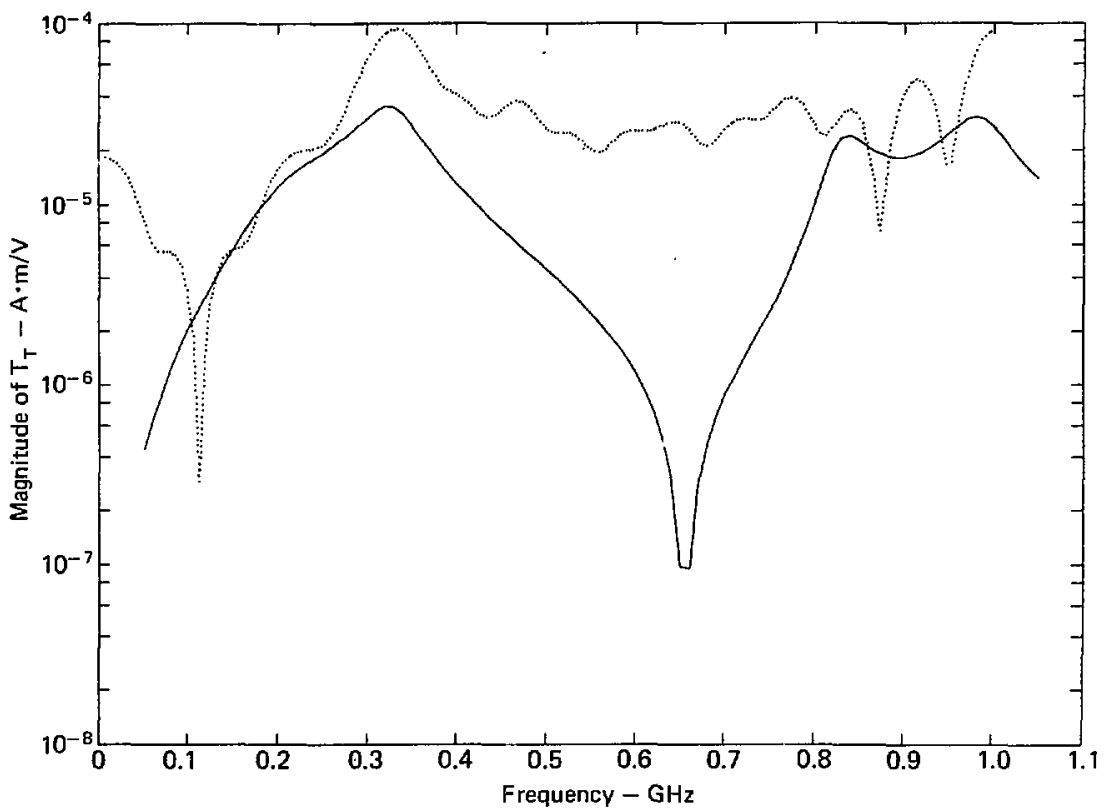

Plot 41. Same graph as Plot 33, except the aximl and circumferential dimensions of the aperture are 0.1 and $0.15 \mathrm{~m}$, respectively. The aperture is on the shaded side of the cylinder. 


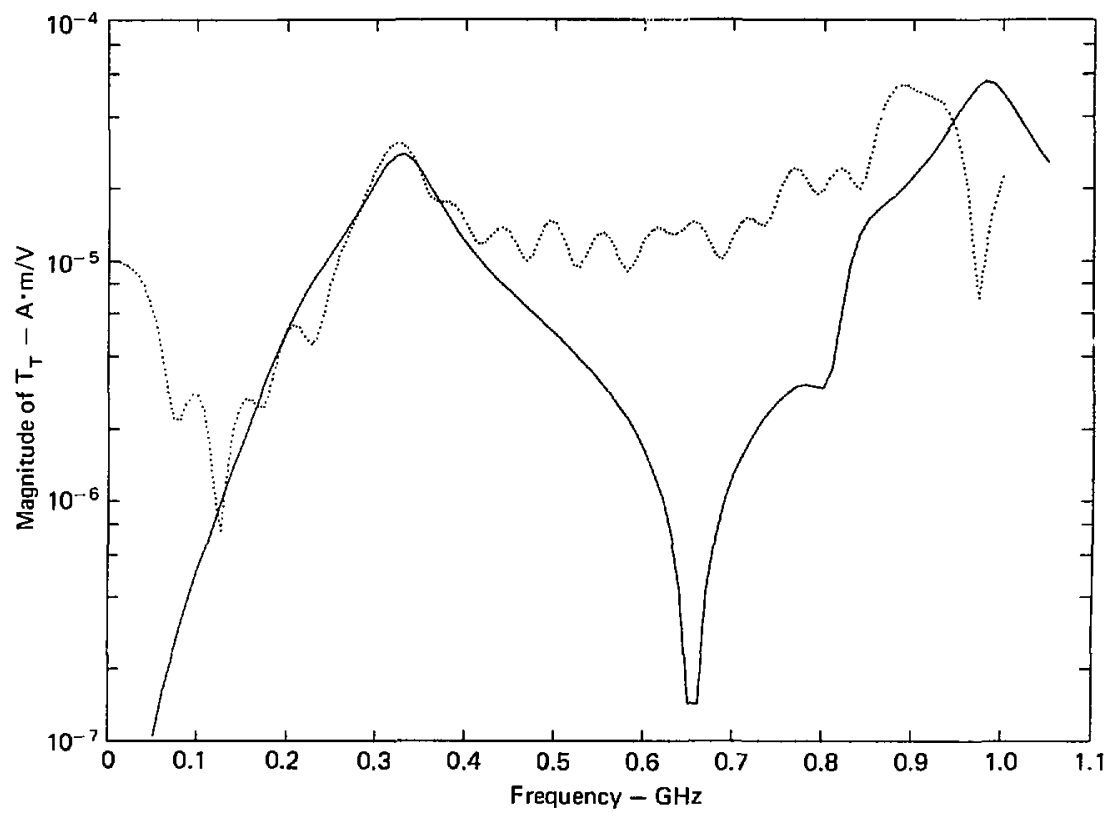

Plot 42. Same graph as Plot 33, except the axjal and circumferential dimensions of the aperture are 0.3 and $0.05 \mathrm{~m}$, respectively. The aperture is on the illuminated side of the cylinder. 


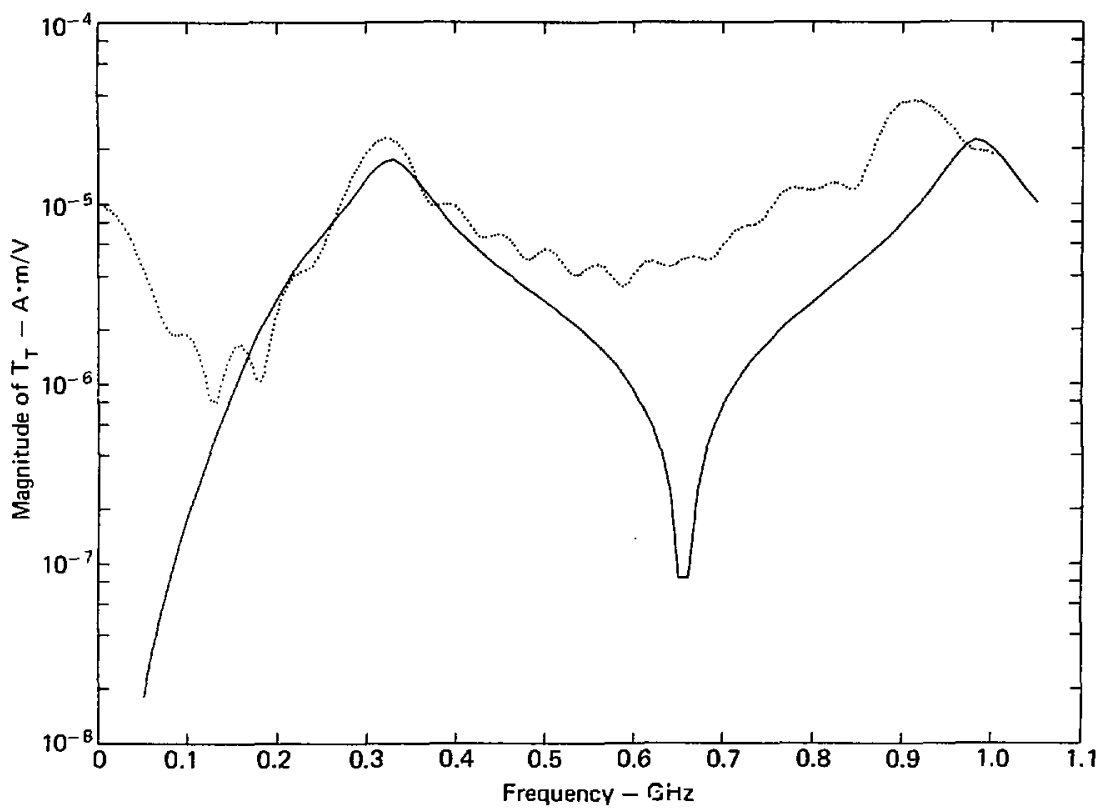

Plot 43. Same graph as Plot 33, except the axial circumferential dimensions of the aperiures are 0.3 and 0.05 m, respectively. The apertures is on the slde of the cylinder that is $90^{\circ}$ from head-an incidence. 


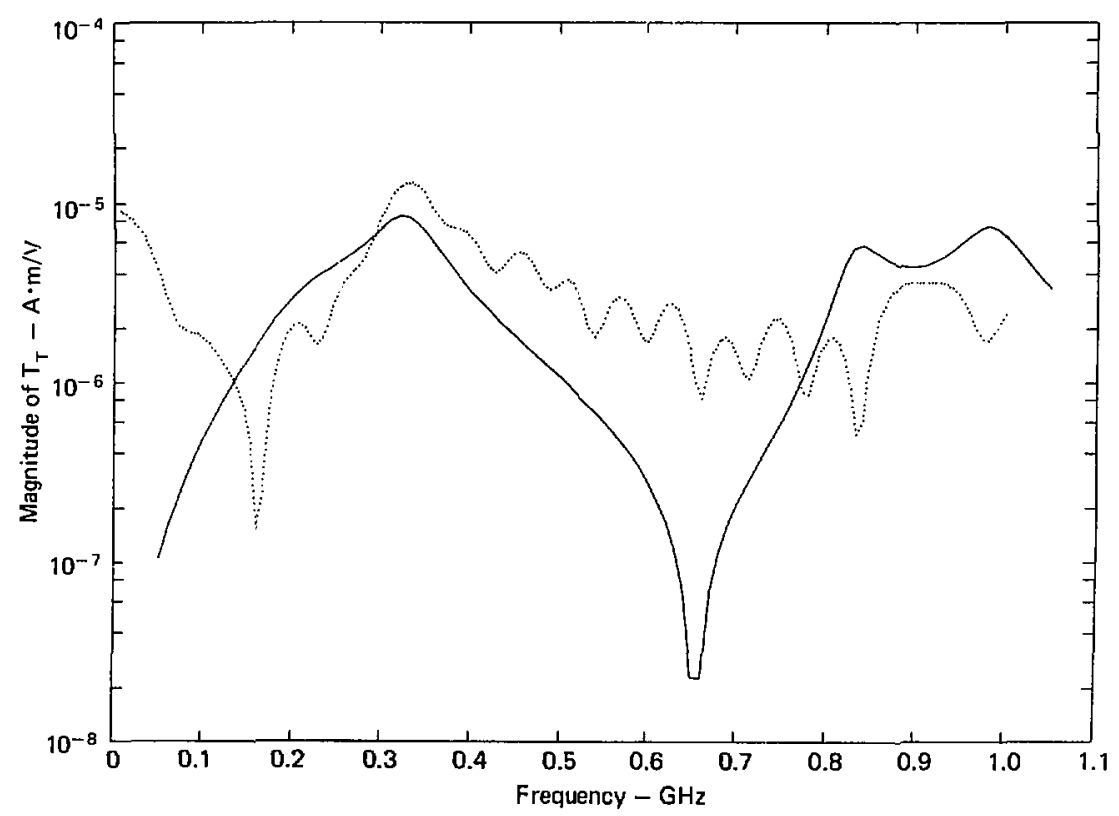

Plot 44. Same graph as Plot 33, except the axial and clrcumferential dimensions of the aperture are 0.3 and 0.05 in, respectively. The aperture is on the shaded skde of the cylinder. 


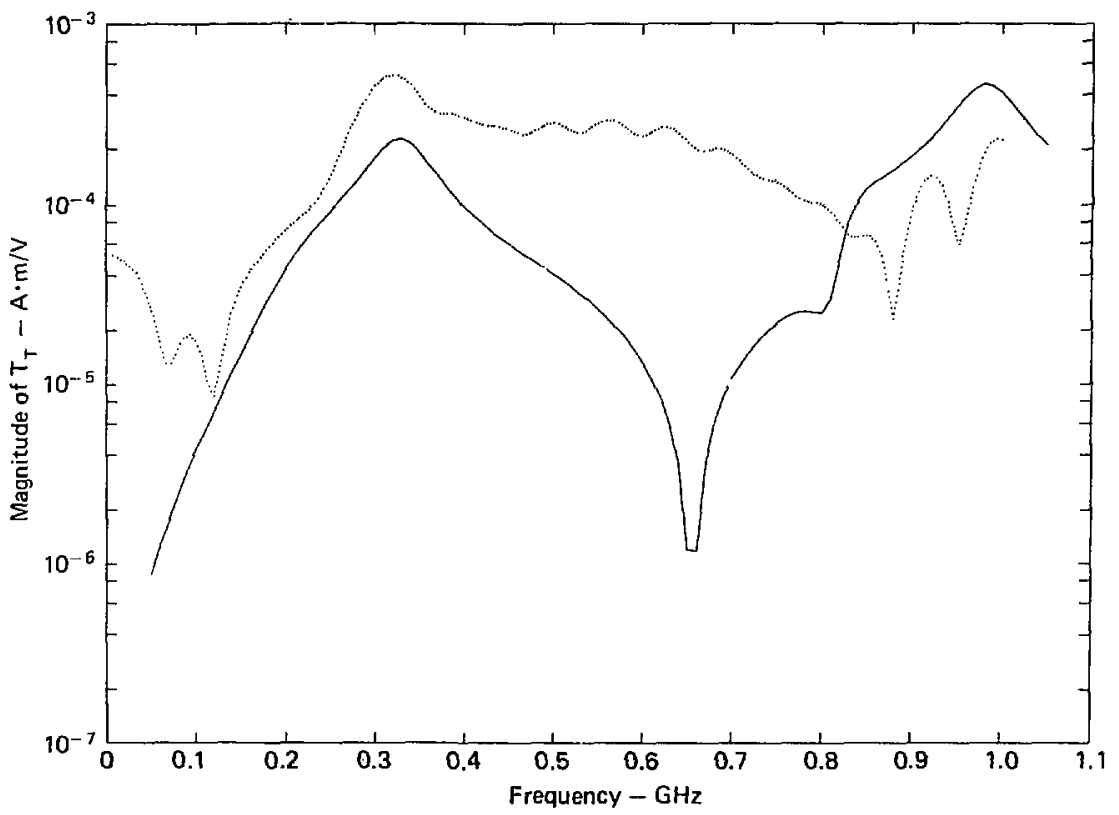

Plot 45. Same graph as Plot 33, except the axial and circumferential dimensions of the aperture arc 0.1 and $0.2 \mathrm{~m}$, respectixcly. The aperture is on the illuminated side of the cylinder. 


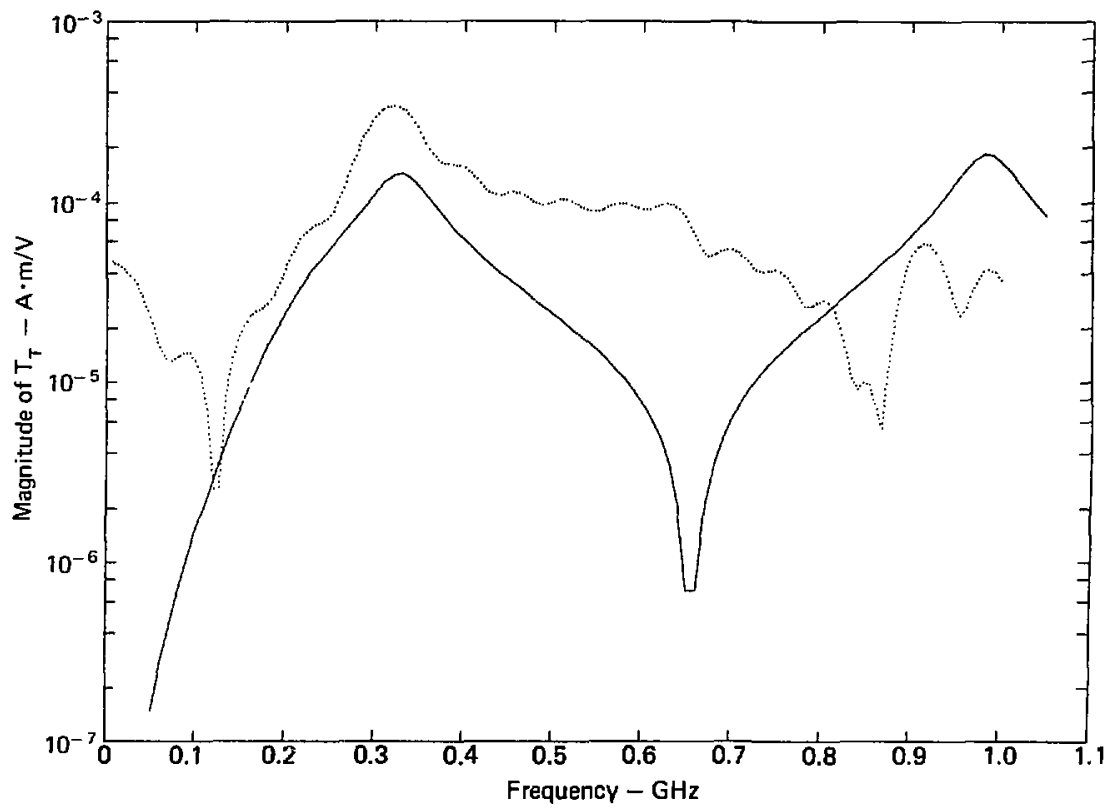

Plot 46. Same graph as Plot 33, except the axinl and circumferential dimensions of the aperture are 0.1 and $0.2 \mathrm{~m}$, respectively. The aperture is on the side of the cylinder that is $90^{\circ}$ from head-on incidence. 


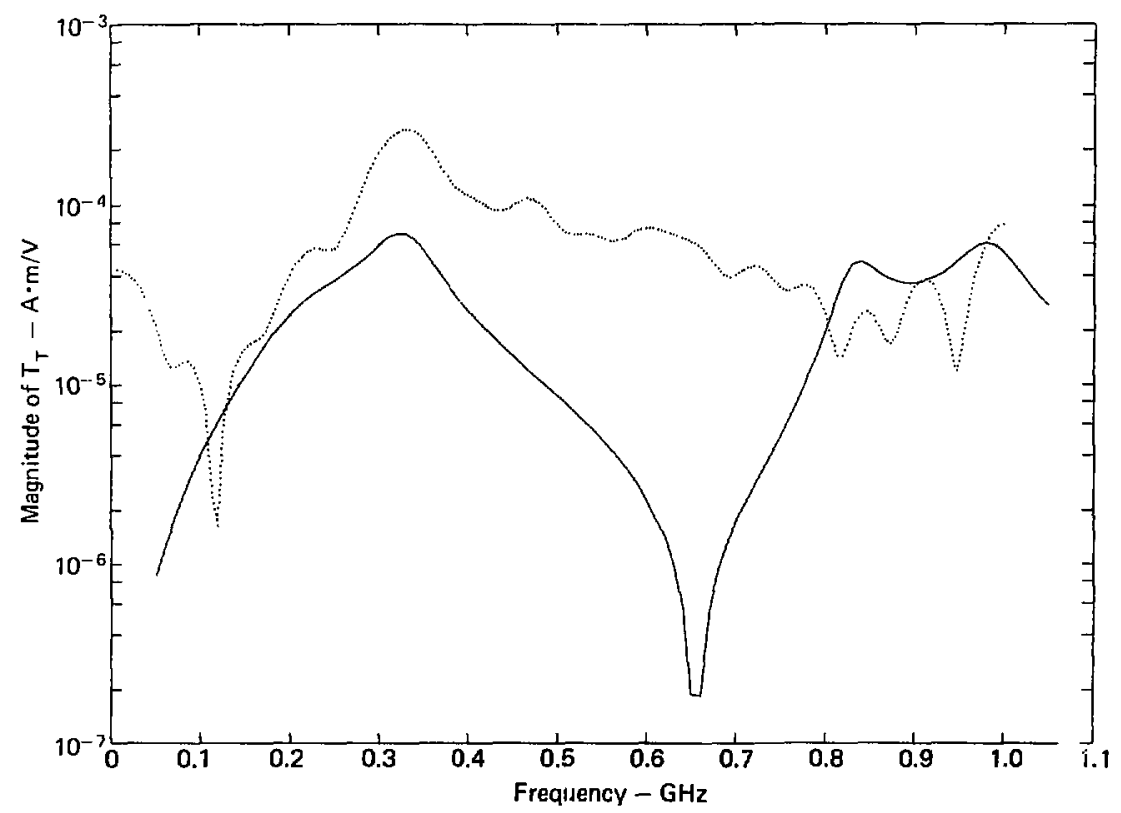

Plot 47. Same graph as Plot 33, except the uxisl end circumferemial dimensions of the aperture are 0.1 mnd $0.2 \mathrm{~m}$, respectively. The aperture is on the shaded side of the cylinder. 


\section{DISCUSSION OF RESULTS}

The magnitude of the current on the exterior of the cylind'r calculated by adding the thin-wire solution from $\mathrm{NEC}^{4}$ to the magnetostatic term developed by Sancer ${ }^{5}$ has the expected functional form. This normalized cursent density is an incident plane wave at a point on the illuminated side, $90^{\circ}$ from head-on incidence, and shaded side of the cylinder shown in Plots 1-3. The first resonance of ihese curves occurs at the frequency for the wire and its image. Note that the magnetostatic current in Eq. (3) has a maximum on the illuminated side of the cylinder but is zero on the side of the cylinder that is $90^{\circ}$ from head-on incidence. Therefore, a measure of the effect of adding the magnetostatic current to thin wire solutions can be obtained by comparing the first peak values on Plots 1 and 2 . The peak on the first plot is about $14 \%$ higher than the peak on the curve. Therefore, the magnetostatic term has some effect on the current on wire, but this effect is minjmal. Plot 4 shows the normalized magnitude of the current at the base of tile wire resulting from a unit-dipole magnetic-current source on the inner surface of the cylinder. These calculations show the normal peak and nulls of a conventional transmission line.

The normalized magnitude of the current at the end of the wire from an incident plane wave is shown as a function of frequency in Plots 5-13. Both the numerical and experimental curves are shown in these plots. The numerically generated data have three peaks and deep nulls in the frequency range shown in these plots. The experimental data for the 0.02 -by $-0.01-\mathrm{m}$ aperture show the second and third peak present in the numerical data but do not contain the first peak. These experimental curves fail to have the deep nulls that are present in the numerical data. The experimental data for the 0.06-by-0.01-m aperture show the three peaks that are present in the numerical model, but not all of the nulls are present. The experimental data for the $90^{\circ}$ circumferential slit have the three peaks and nulls that are present in the numerical data. Note that in all of these data there is a rather large ripple in curves as the frequency is varied. This ripple is caused by the fast Fourier transform. Since the usable time window of the transient measurement range set the total time that data can be obtained, the fast Fourier transform has only a fixed time window available to find the frequency response of the measured current. If the time function does not sufficiently approach zero in that given time window, then the effect of truncating the signal can result in a ripple in the frequency response. This truncation ripple can obscure the presence of both peaks and nulls in the data and is probably the cause of some slight difference between the measured and calculated results.

In the data for the first two apertures in the thin cylinder, the experimental and numerical magnitudes are very closely related. This statement is true when limitation of the ripple in the data is overlooked. On the other hand, the experimental data are two orders of magnitude less than the numerical data for the curve of the $90^{\circ}$ circumferential slot in a thin cylinder. This effect on the circumferential slot can be attributed to the current on the surface of the cylinder. The current on the cylinder can be written as the sum of two currents, one being the unperturbed current on the cylinder before the aperture is cut into the cylinder, while the second is the current describing the amount the first current changes when the aperture is cut into the cylinder. The numerical calculations are performed with the unperturbed current. In the case of the first apertures used in thin cylinders, the perturbed current term must be very small. In the case of $90^{\circ}$ circumferential slot, the presence of the aperture greatly changes the current on the cylinder resulting in a great change in the magnetic field that couples into the inside of the aperture.

Plots 14-28 display the experimental and numerical data for the normalized magnitude of the current at the base of the wire behind an aperture in a plane resulting from an incident plane wave. The numerical data show the normal transmission tine peaks and nulls as the frequency is increased. The experimental data display the first of two peaks that are present in the numerical data. The values of these experimental peaks are less than those in the numerical data. The experimental data in these studies also contain ripple as did the data for the thin cylinder. As noted above, this ripple is caused by the fast Fourier transform and the limited time window of the transient range. This ripple is probably the cause of some slight difference between the measured and calculated results. Note that, in all the plots except two, the low frequency experimental data have adequate agreement with the numerical data. The low frequency experimental data presented in Plots 21 and 24 do not agree with the numerical data. We may conelude from this that the experimental data given on Plots 21 and 24 are 
incorrect due to measurement errors on the range. One possible source of the error may be a poor electrical connection bet $y$ 'een the wire behind the aperture and the electrical connector in which the wire was placed.

All the experimental data for the wire behind a plane are slightly less than the numerical data for low frequency. As the frequency increases, the difference between the experimental and numerical data increases. This increase can be attributed to the fact that more energy from the aperture radiates past the wire as the frequency is increased. As the frequency is increased, the distance in wave length between a wirc and the aperture increases. This increase in the wire-aperture distance means an increase in the energy that radiates past the wire, which in turn means less current on the wire. On the other hand, the numerical model assumes that all of the energy from the aperture goes into transverse electromagnetic (TEM) modes on the wire.

The normalized magnitude of the current density on the outside surface of a fat cylinder excited by an incident plane is plotted as a function of frequency in Plots 29-31. The first resonance shows that these data correspond to free-space wavelengths of twice the distance from the center of the upper cylinder cap to the center of the bottom cap for the case of cylinder and its image through the ground plane. The normalized magnitude of current at the base of the wire excited by a unit magnetic dipole source on the inside surface of the cylinder is shown in Plot 32. This curve has the standard peaks and nulls of a transmission line.

Plots 33-47 display the normalized magnitude of the current at the bottom of a wire behind an aperture in a fat cylinder excited by the incident plane wave. In each of these figures, both the numerical and experimental results are displayed as functions of frequency. The numerical data presented in these curves have two peaks and a deep null in the frequency range shown. All of the experimental data have a peak that corresponds to the first peak of the numerical data, while only some of the experimental data exhibit the second peak. The deep nulls are missing from the experimental data. These experimental data also have ripple that can be attributed to the fast Fourier transform and the limited time window of the transient range. Note that the wire's clirrent should decrease as the aperture is moved from the illuminated to the shaded side of the cylinder. Comparing Plot 31 with Plot 33 leads to only one conclusion: the experimental data given in Plot 33 are incorrect. One possible source of this problem may be a poor electrical connection between the base of the wire and the sampling scope.

In all of the data presented for a fat cylinder, the magnitude of the current measured experimentally is greater than currents calculated numerically. The magnitude of the first peak for the experimental data ranges from 0 to 6 times the first peak for the numerical data. The reason for the numerical model's failure to predice the experimental results probably can be attributed to the limitation of the Bethe hole theory. As noted above, the Bethe hole theory was developed for an electrically small aperture in a flat surface. In this study, the theory was used to model large apertures in a curved surface. There might be two possible explanations for the failure of the modeling procedure. The modeling of apertures on a cylinder by a planar approximation might predict too little energy coupling through the aperture. The second explanation could be that the modeling of large apertures by Bethe hole theory might also lead to a prediction of too little energy coupling through apertures. These two possible failures of Bethe hole theory need investigation in further work.

\section{FUTURE WORK}

This work investigated the applicability of Bethe hole theory for numerical modeling an aperture in a cylinder and a plane with a wire behind the aperture. A comparison of numerical and experimental results indicated adequate agreement for the case of the thin cylinder, but failed for the cases of the thick cylinder and plane. Due to several assumptions made by Taylor and Harrison ${ }^{2}$ in the development of the method used to model apertures in cylinders, their results are limited. Two such limitations are that the apertures must not be near the end of the cylinder and the internal wire can only be along the axis of the cylinder. There are no ways to modify these assumptions in the development of this method. These assumptions severely limit the usefulness of the method when considering practical EMP coupling problems. The general electromagnetic coupling from the exterior to the interior on large, irregular, three-dimensional bodies through apertures is an un resolved problem.

To improve the numerical modeling of apertures in three-dimensional body, an effort must be made 
to improve the present moment-method codes for this problem. An improved moment-method capability would give an insight into many practical aperture problems. But the moment-method approach would fail for large problems due to the limitation of the size of the matrix that can presently be stored and solved by a computer. A method that does not have this problem is the finite-difference solution. The finite-difference solution has a banded matrix, therefore requires less time to solve on a computer than the moment-method solution. The speed of the finite-difference solution is bought at the cost of the accuracy of the numerical model. The moment-method approach works best for thinwires and apertures while the finite-difference methods works best for complicated threedimensional bodies. To take advantage of the strengths of these two methods a new hybrid finitedifference-moment-method approach would be useful. This approach would combine the momentmethod approach to a finite-difference solution into one numerical code. This code would ailow different regions of the problem to be handled by the appropriate methods. The development of this new hybrid method would appear to be possible from our limited investigation. A new hybrid code would provide a method to calculate a large class of complex practical problems.

Numerical methods can be used to model many practical problems, but there are some very large, complex EMP problems that can be handled only by experimental measurements. A systematic collection of experimental data would be useful. The experimental data would supplement the computational ability of numerical modeling. This experimental data would be found for a large class of canonical problems that represent most practical external-internal coupling geometries. Some of these geometries are wires behind apertures in rectangular and cylindrical shells. In this data set the effect of varying the size, shape, and position of both the apertures and wires would be needed. Also, the effects of cavities backing the aperture would have to be included. The data would be presented in a format that is user-oriented. With the hybrid computer code and all the necessary experimental data, any practical EMP-aperture problem could be treated.

\section{SUMMARY}

From this study we have learned several facts about the modeling of apertures:

- The Bethe hole theory coupling into a TEM mode of a wire at the center of an electrically thin cylinder gives reasonable results when compared with experimental measurements.

- The Bethe hole theory coupling into a TEM mode of a wire behind an aperture in a plane gives values that are greater than those measured by experimental measurements.

- The Bethe hole theory coupling into a TEM mode for a wire at the center of an electrically large cylinder gives numerical results that are less than the experimental measurements.

- The addition of the magnetostatic term hes a minimal effect on the calculation of the current on a wire in the cylinder.

- Bethe hole theory does not work for electrically large apertures.

- In the case of a wire behind ar: aperture in a plane, an assumption was made that all the cnergy is carried away from the aperture by the TEM mode on the wire. This assumption does not allow for a significant amount of energy that radiates past the wire.

\section{ACKNOWLEDGMENTS}

This work was performed for the Defense Nuclear Agency under DNA Subtask R99.
QAXEB088 Work Unit 76: Experimental Coupling Model Verification for Generic Structures. 


\section{REFERENCES}

1. J. N. Brittingham, A Literature Review of EMP Effects on Apertures, Lawrence Livermore Laboratory, Livermore, CA, UCID-17321 (1976).

2. C. D. Taylor and C. W. Harrison, "On the Excitation of a Coaxial Line Through a Small Aperture in the Outer Sheath," Air Force Weapons Laboratory, Kirtland AFB, NM, Air Force Weapons Laboratory $\mathbf{l n}^{\mathrm{n}}$ teractir Notes \#104 (1972).

3. R. E. Collin, Field Theory of Guided Waves (McGraw-Hill Book Company, New York, 1960), pp. $295-$ 298.

4. G. J. Burke and A. J. Poggio, Numerical Electromagnetic Code-Moment Method, Naval Ocean Systems Center, San Diego, CA, NOSC/TD 116 (1977).

5. M. I. Sancer, Fundamental Errors Associated with the Gross Modeling of the Physical Features of Metallic Enclosures, Air Force Weapons Laboratory, Kirtland AFB, NM, Air Force Weapons Laboratory Interaction Notes \#289 (1976).

6. D. Kajfez, Excitation of a Terminated TEM Transmission Line Through a Small Aperture, Air Force Weapons Laboratory, Kirtland AFB, NM, Air Force Weapons Laboratory Interaction Note \#215 (1974).

7. F. J. Deadrick, E. K. Miller, and H. G. Hudson, $7 \%_{\mathrm{k}}$ I.LL Transient Electromagnetic Measurement Facility, Lawrence Livermore Laboratory, Livermore, CA, UCRL-51933 (1975). 